DOI 10.4171/JEMS/351

Monica Musso · Frank Pacard · Juncheng Wei

\title{
Finite-energy sign-changing solutions with dihedral symmetry for the stationary nonlinear Schrödinger equation
}

Received January 21, 2009 and in revised form February 23, 2010, December 14, 2010 and January 9,2011

Abstract. We address the problem of the existence of finite energy solitary waves for nonlinear Klein-Gordon or Schrödinger type equations $\Delta u-u+f(u)=0$ in $\mathbb{R}^{N}, u \in H^{1}\left(\mathbb{R}^{N}\right)$, where $N \geq 2$. Under natural conditions on the nonlinearity $f$, we prove the existence of infinitely many nonradial solutions in any dimension $N \geq 2$. Our result complements earlier works of Bartsch and Willem [1] ( $N=4$ or $N \geq 6)$ and Lorca and Ubilla [13] $(N=5)$ where solutions invariant under the action of $O(2) \times O(N-2)$ are constructed. In contrast, the solutions we construct are invariant under the action of $D_{k} \times O(N-2)$ where $D_{k} \subset O(2)$ denotes the dihedral group of rotations and reflections leaving a regular planar polygon with $k$ sides invariant, for some integer $k \geq 7$, but they are not invariant under the action of $O(2) \times O(N-2)$

Keywords. Nonradial bound states, nonlinear Schrödinger equations, balancing condition, Lyapunov-Schmidt reduction method

\section{Introduction and statement of the main results}

Nonlinear semilinear elliptic equations of the form

$$
\Delta u-u+f(u)=0 \quad \text { in } \mathbb{R}^{N},
$$

$u \in H^{1}\left(\mathbb{R}^{N}\right)$, arise in various models in physics, mathematical physics and biology. In particular, the study of standing waves (or solitary waves) for the nonlinear Klein-Gordon or Schrödinger equations reduces to (1.1). We refer to the papers of Berestycki and Lions [3], [4] and Bartsch and Willem [1] for further references and motivations.

Obviously (1.1) is equivariant with respect to the action of the group of isometries of $\mathbb{R}^{N}$, it is henceforth natural to ask whether all solutions of (1.1) are radially symmetric. In that regard, the classical result of Gidas, Ni and Nirenberg [7] asserts that all

M. Musso: Departamento de Matemática, Pontificia Universidad Catolica de Chile, Avda. Vicuña Mackenna 4860, Macul, Chile; e-mail: mmusso@mat.puc.cl

F. Pacard: Centre de Mathématiques Laurent Schwartz, UMR-CNRS 7640, École Polytechnique, 91128 Palaiseau, France and Institut Universitaire de France; e-mail: frank.pacard@ math.polytechnique.fr

J. Wei: Department of Mathematics, The Chinese University of Hong Kong, Shatin, Hong Kong; e-mail:wei@math.cuhk.edu.hk

Mathematics Subject Classification (2010): 35J25, 35J20, 35B33, 35B40 
positive solutions of (1.1) are indeed radially symmetric. Therefore, nonradial solutions, if they exist, are necessarily sign-changing. When the nonlinearity $f$ is odd, Berestycki and Lions [3], [4] and Struwe [16] have obtained the existence of infinitely many radially symmetric sign-changing solutions under some (almost necessary) growth condition on $f$ (we also refer to the work of Bartsch and Willem [2] and Conti, Merizzi and Terracini [5] for different approaches and weaker assumptions on the nonlinearity $f$ ).

The existence of nonradial sign-changing solutions was first proved by Bartsch and Willem [1] in dimension $N=4$ and $N \geq 6$. The key idea is to look for solutions invariant under the action of $O(2) \times O(N-2) \subset O(N)$ to recover some compactness property. Later on, this result was generalized by Lorca and Ubilla [13] to handle the $N=5$-dimensional case. The proofs of both results rely on variational methods and the oddness of the nonlinearity $f$ is needed. The question of the existence of nonradial solutions remained open in dimensions $N=2,3$.

In this paper, we construct unbounded sequences of solutions of (1.1) in any dimensions $N \geq 2$. The solutions we obtain are nonradial, have finite energy and are invariant under the action of $D_{k} \times O(N-2)$, for some given $k \geq 7$, where $D_{k} \subset O(2)$ is the dihedral group of rotations and reflections leaving a regular polygon with $k$ sides invariant. Moreover, these solutions are not invariant under the action of $O(2) \times O(N-2)$ and hence they are different from the solutions constructed in [1] and [13].

We set

$$
u_{+}:=\max (u, 0) \quad \text { and } \quad u_{-}:=\max (-u, 0) .
$$

We will assume that the nonlinearity $f$ can be decomposed as

$$
f(u)=f_{1}\left(u_{+}\right)-f_{2}\left(u_{-}\right),
$$

where the functions $f_{i}: \mathbb{R} \rightarrow \mathbb{R}$ are at least $\mathcal{C}^{1, \mu}$ for some $\mu \in(0,1)$ and satisfy the following conditions:

(H.1) For $i=1,2, f_{i}(0)=f_{i}^{\prime}(0)=0$.

(H.2) For $i=1,2$, the equation

$$
\Delta w_{i}-w_{i}+f_{i}\left(w_{i}\right)=0
$$

has a unique positive (radially symmetric) solution $w_{i}$ which tends to 0 exponentially fast at infinity.

(H.3) For $i=1,2$, the solution $w_{i}$ is nondegenerate, in the sense that

$$
\operatorname{Ker}\left(\Delta-1+f_{i}^{\prime}\left(w_{i}\right)\right) \cap L^{\infty}\left(\mathbb{R}^{N}\right)=\operatorname{Span}\left\{\partial_{x_{1}} w_{i}, \ldots, \partial_{x_{N}} w_{i}\right\}
$$

A typical example of a nonlinearity $f$ satisfying all the above assumptions is the function

$$
f(u)=\left(u_{+}^{p_{1}}-c_{1} u_{+}^{q_{1}}\right)-\left(u_{-}^{p_{2}}-c_{2} u_{-}^{q_{2}}\right),
$$

where $c_{i} \geq 0$ and $1<q_{i}<p_{i}<(N+2) /(N-2)$ (we agree that $(N+2) /(N-2)=+\infty$ when $N=2$ ). In this case, the existence of $w_{i}$ is standard and follows from well known 
arguments in the calculus of variation while the uniqueness follows from the results of Kwong [10] and Kwong and Zhang [11]. Concerning the nondegeneracy condition (which essentially follows from the uniqueness of the solutions), we refer to Appendix C of [15].

For example, when $p_{1}=p_{2}=p$ and $c_{1}=c_{2}=0$, the nonlinearity is just given by

$$
f(u)=|u|^{p-1} u \text {. }
$$

The energy functional associated to (1.1) is given by

$$
\mathcal{E}(u):=\frac{1}{2} \int_{\mathbb{R}^{N}}\left(|\nabla u|^{2}+u^{2}\right) d x-\int_{\mathbb{R}^{N}} F(u) d x,
$$

where

$$
F(u):=\int_{0}^{u} f(s) d s
$$

We will denote by $\mathcal{E}_{i}$ the energy of the function $w_{i}$,

$$
\mathcal{E}_{i}:=\frac{1}{2} \int_{\mathbb{R}^{N}}\left(\left|\nabla w_{i}\right|^{2}+w_{i}^{2}\right) d x-\int_{\mathbb{R}^{N}} F\left(w_{i}\right) d x .
$$

Given the above notation and definitions, we can now state the main result of this paper.

Theorem 1.1. Assume that the nonlinearity $f$ satisfies the assumptions (H.1)-(H.3) and that $k \geq 7$ is a fixed integer. Then there exist two sequences of integers, $\left(m_{i}\right)_{i \geq 0}$ and $\left(n_{i}\right)_{i \geq 0}$, tending to $+\infty$, and $\left(u_{i}\right)_{i \geq 0}$, a sequence of nonradial sign-changing solutions of (1.1), whose energy $\mathcal{E}\left(u_{i}\right)$ is equal to

$$
\mathcal{E}\left(u_{i}\right)=k\left(\left(m_{i}+n_{i}\right) \mathcal{E}_{1}+n_{i} \mathcal{E}_{2}\right)+o(1) .
$$

Moreover, the solutions $u_{i}$ are invariant under the action of $D_{k} \times O(N-2)$ but are not invariant under the action of $O(2) \times O(N-2)$.

Observe that we do not assume that the function $f$ is odd, and hence oddness of the nonlinearity is not a necessary condition for the existence of nonradial solutions of (1.1). The assumption (H.3) on the nonlinearity $f$ reflects the techniques we use: instead of variational methods, we are going to use singular perturbation techniques to prove Theorem 1.1. This might look rather counterintuitive since, in most singularly perturbed problems, a small parameter is needed (generally it appears as a coefficient in front of the Laplacian or in front of the nonlinearity) in order to ensure that an appropriate sequence of functions constitute good enough approximate solutions as the parameter tends to its limit value (generally 0 ).

There is no such small parameter in (1.1). Instead, we use the noncompactness of the space of finite energy solutions of (1.1) to build a discrete sequence of functions which are as close to being solutions as desired. The idea is to consider a regular polygon with $k$ sides and very large radius. Along each of the $k$ rays joining the origin to the vertices of the polygon, we arrange $m$ copies of the entire positive solution $w_{1}$ at distance $\ell \gg 1$ from each other and, along each of the $k$ sides of the polygon, we arrange alternatingly $n$ 
copies of the entire positive solution $w_{1}$ with $n$ copies of the entire negative solution $-w_{2}$ at distance $\bar{\ell} \gg 1$ from each other. As $\ell$ and $\bar{\ell}$ tend to infinity (and hence the radius of the regular polygon tends to infinity), the corresponding function is close (in a sense to be made precise) to being a solution of (1.1). We will adjust the discrete parameters $m, n$ and the continuous parameters $\ell$ and $\bar{\ell}$, which determine the location of the points where the solutions $w_{1}$ and $-w_{2}$ are centered, so that some global equilibrium is achieved, and this will imply that the approximate solution can be perturbed to a genuine solution of (1.1). A similar idea has already been used by Wei and Yan in [17] where infinitely many positive bound states for a class of nonlinear Schrödinger equations are constructed. But, in our case, the intuition for our construction certainly comes from a similar construction which has been obtained by Kapouleas in the context of compact constant mean curvature surfaces of Euclidean 3-space [9]. We shall briefly discuss this at the end of this section.

It turns out that the sequences of integers, $\left(m_{i}\right)_{i \geq 0}$ and $\left(n_{i}\right)_{i \geq 0}$, which appear in the statement of Theorem 1.1 are not arbitrary and in fact they are related by some nonlinear equation. To explain this, we need to introduce what we call the interaction function $\Psi_{i \rightarrow j}$ which is defined for all $s \in \mathbb{R}$ by

$$
\Psi_{i \rightarrow j}(s):=-\int_{\mathbb{R}^{N}} w_{i}(x-s e) \operatorname{div}\left(f_{j}\left(w_{j}(x)\right) e\right) d x,
$$

where $e \in \mathbb{R}^{N}$ is any unit vector and $i, j \in\{1,2\}$. It is easy to check that this definition is independent of the choice of e, and hence it only depends on $s>0$. Indeed, if $R \in O(n)$, using the fact that $w_{1}$ and $w_{2}$ are radially symmetric, we can write

$$
\int_{\mathbb{R}^{N}} w_{i}(x-s e) \operatorname{div}\left(f_{j}\left(w_{j}(x)\right) e\right) d x=\int_{\mathbb{R}^{N}} w_{i}(R(x-s e)) \operatorname{div}\left(f_{j}\left(w_{j}(R x)\right) e\right) d x,
$$

and, performing a change of variables, we conclude that

$$
\int_{\mathbb{R}^{N}} w_{i}(x-s e) \operatorname{div}\left(f_{j}\left(w_{j}(x)\right) e\right) d x=\int_{\mathbb{R}^{N}} w_{i}(x-s R e) \operatorname{div}\left(f_{j}\left(w_{j}(x)\right) R e\right) d x
$$

for all $R \in O(n)$.

With this notation at hand, the sequences $m_{i}$ and $n_{i}$ are related by

$$
\left(2 \sin \frac{\pi}{k}\right) m_{i} \ell_{i}=2 n_{i} \bar{\ell}_{i}-\bar{\ell}_{i}^{\prime},
$$

where the real numbers $\ell_{i}, \bar{\ell}_{i}, \bar{\ell}_{i}^{\prime}>0$ have to be large enough and satisfy

$$
\Psi_{1 \rightarrow 1}\left(\ell_{i}\right)=\left(2 \sin \frac{\pi}{k}\right) \Psi_{2 \rightarrow 1}\left(\bar{\ell}_{i}\right) \quad \text { and } \quad \Psi_{1 \rightarrow 1}\left(\ell_{i}\right)=\left(2 \sin \frac{\pi}{k}\right) \Psi_{1 \rightarrow 1}\left(\bar{\ell}_{i}^{\prime}\right) .
$$

We shall further comment on the solvability of this system of equations at the end of this section.

Finally, let us mention that Malchiodi [14] has recently constructed positive (infinite energy) solutions of (1.1) by perturbing a configuration of infinitely many copies of the 
positive solution $w_{1}$ arranged along three rays meeting at a common point. The solutions he has constructed are bounded but have infinite energy. Our key observation is that solutions with finite energy can be obtained using similar ideas provided one considers signchanging solutions, and this is precisely the contribution of our paper. Let us also mention that positive solutions of (1.1) with unbounded energy have also been constructed by del Pino, Kowalczyk, Pacard and Wei in [6] again using ideas which stem from a similar construction in the theory of noncompact constant mean curvature surfaces of Euclidean 3-space.

The proof of the main result is rather technical and, in order to help alleviate the complexity of the notation and present the main ideas as clearly as possible, we will prove Theorem 1.1 in the case where the nonlinearity is given by

$$
f(u)=|u|^{p-1} u \text {. }
$$

Mutatis mutandis, the proof goes through for any nonlinearity satisfying (H.1)-(H.3). Therefore, from now on, we will be interested in solutions of

$$
\Delta u-u+|u|^{p-1} u=0 \quad \text { in } \mathbb{R}^{N}
$$

which tend to 0 as $|x|$ tends to $\infty$. We will assume that the exponent $p$ satisfies $1<p<$ $(N+2) /(N-2)$ when $N \geq 3$ and $1<p<+\infty$ when $N=2$. Observe that equation (1.8) is the Euler-Lagrange equation of the functional defined by

$$
\mathcal{E}(u):=\frac{1}{2} \int_{\mathbb{R}^{N}}\left(|\nabla u|^{2}+u^{2}\right) d x-\frac{1}{p+1} \int_{\mathbb{R}^{N}}|u|^{p+1} d x,
$$

and let us recall that there exists a unique radially symmetric (in fact radially decreasing) positive solution of

$$
\Delta w-w+w^{p}=0 \quad \text { in } \mathbb{R}^{N}
$$

which tends to 0 as $|x|$ tends to $\infty$. Moreover, all positive solutions of (1.8) which tend to 0 at $\infty$, are translates of $w$. The function $w$ together with its translations will constitute the building blocks of our construction.

As far as the asymptotic behavior of $w$ at infinity is concerned, it is known that there exists a constant $c_{N, p}>0$, only depending on $N$ and $p$, such that

$$
\lim _{r \rightarrow \infty} e^{r} r^{(N-1) / 2} w=c_{N, p}>0, \quad \text { and } \quad \lim _{r \rightarrow \infty} \frac{w^{\prime}}{w}=-1,
$$

where we have set $r:=|x|$. Of importance to us will be the interaction function $\Psi$ defined by

$$
\Psi(s):=-\int_{\mathbb{R}^{N}} w(\cdot-s e) \operatorname{div}\left(w^{p} e\right) d x,
$$

where $e \in \mathbb{R}^{N}$ is any unit vector. It is known (see Lemma 5.1) that

$$
\Psi(s)=C_{N, p} e^{-s} s^{-(N-1) / 2}\left(1+\mathcal{O}\left(s^{-1}\right)\right) \quad \text { as } s \rightarrow \infty,
$$


where the constant $C_{N, p}>0$ only depends on $N$ and $p$. Similar estimates hold for the derivatives of $\Psi$, and in particular we have

$$
-(\log \Psi)^{\prime}(s)=1+\frac{N-1}{2 s}+\mathcal{O}\left(s^{-2}\right) \quad \text { as } s \rightarrow \infty .
$$

Finally, the solution $w$ is nondegenerate in the sense defined in (1.3) (we refer the reader to [15] for a proof of this fact).

Recall that being nondegenerate is equivalent to the fact that the $L^{\infty}$-kernel of the operator

$$
L_{0}:=\Delta-1+p w^{p-1},
$$

which is nothing but the linearized operator about $w$, is spanned by the functions

$$
\partial_{x_{1}} w, \ldots, \partial_{x_{N}} w
$$

which naturally belong to this space. This nondegeneracy property will be crucial in our construction.

As already mentioned, the solutions we construct are invariant under a large group of symmetries. More precisely, they will enjoy the following invariance:

$$
u(x)=u(R x) \quad \text { for all } R \in\left\{I_{2}\right\} \times O(N-2),
$$

and also

$$
u\left(R_{k} x\right)=u(x) \quad \text { and } \quad u(\Gamma x)=u(x),
$$

where $R_{k} \in O(2) \times\left\{I_{N-2}\right\}$ is the rotation of angle $2 \pi / k$ in the $\left(x_{1}, x_{2}\right)$-plane and $\Gamma \in$ $O(2) \times\left\{I_{N-2}\right\}$ is the symmetry with respect to the hyperplane $x_{2}=0$. Here $I_{2}$ and $I_{N-2}$ denote respectively the identity in $\mathbb{R}^{2}$ and in $\mathbb{R}^{N-2}$.

The solutions of (1.8) we construct are small perturbations of the sum of copies of $\pm w$, centered at carefully chosen points in $\mathbb{R}^{2} \times\{0\} \subset \mathbb{R}^{N}$. Let us now give a precise description of these points. We fix an integer $k \geq 7$, which will define the dihedral group we are working with, and we assume that we are given two positive integers $m, n$ and two positive real numbers $\ell, \bar{\ell}$ related by

$$
\left(2 \sin \frac{\pi}{k}\right) m \ell=(2 n-1) \bar{\ell} .
$$

The canonical basis of $\mathbb{R}^{N}$ will be denoted by

$$
e_{1}:=(1,0, \ldots, 0), \quad e_{2}:=(0,1,0, \ldots, 0), \quad \ldots, \quad e_{N}:=(0, \ldots, 0,1)
$$

We consider the inner polygon which is the regular polygon in $\mathbb{R}^{2} \times\{0\} \subset \mathbb{R}^{N}$ whose vertices are given by the points of the orbit of the point

$$
\stackrel{\circ}{y}_{1}:=\frac{\bar{\ell}}{2 \sin \frac{\pi}{k}} e_{1} \in \mathbb{R}^{N}
$$


under the action of the group generated by $R_{k}$. By construction, the edges of this polygon have length $\bar{\ell}$.

We now define the outer polygon which is a regular polygon whose vertices are the points of the orbit of the point

$$
\stackrel{\circ}{y+1}_{m+1}:=\stackrel{\circ}{1}_{1}+m \ell e_{1},
$$

under the group generated by $R_{k}$. Observe that the distance from $\stackrel{\circ}{y+1}_{m}$ to the origin is $m \ell+\frac{\bar{\ell}}{2 \sin (\pi / k)}$ and, thanks to (1.17), the edges of the outer polygon have length $2 n \bar{\ell}$.

By construction, the distance between the points $\dot{\circ}_{1}$ and $\dot{y}_{m+1}$ is equal to $m \ell$ and we will denote by $\stackrel{\circ}{y}_{j}$ for $j=2, \ldots, m$ the points evenly distributed on the segment between these two points

$$
\stackrel{\circ}{y}_{j}:=\stackrel{\circ}{y}_{1}+(j-1) \ell e_{1} \quad \text { for } j=2, \ldots, m .
$$

As already mentioned, the edges of the outer polygon have length $2 n \bar{\ell}$ and hence the distance between $\stackrel{\circ}{y}_{m+1}$ and $R_{k} \stackrel{\circ}{y}_{m+1}$ is $2 n \bar{\ell}$. Again we distribute points $\stackrel{\circ}{z}_{h}, h=$ $1, \ldots, 2 n-1$, evenly along this segment. More precisely, if

$$
t:=-\sin \frac{\pi}{k} e_{1}+\cos \frac{\pi}{k} e_{2} \in \mathbb{R}^{N},
$$

then

$$
\stackrel{\circ}{z}_{h}:=\stackrel{\circ}{y}_{m+1}+h \bar{\ell} t \quad \text { for } h=1, \ldots, 2 n-1 .
$$

Observe that, by construction,

$$
R_{k} \stackrel{\circ}{y}_{m+1}=\stackrel{\circ}{y}_{m+1}+2 n \bar{\ell} t .
$$

Remark 1.1. In the general case, when the nonlinearity is not odd and hence $w_{1} \neq$ $w_{2}$, some changes are needed in the definitions of the inner and outer polygons. In the construction of the inner polygon, $\bar{\ell}$ used in (1.18) has to be replaced by $\bar{\ell}^{\prime}$ which is defined in terms of $\ell$ by the second equation in (1.7) and $\bar{\ell}$ which is used to define the outer polygon is defined by the first equation. Finally, (1.17), which relates $\ell, \bar{\ell}$ and $\bar{\ell}^{\prime}$, has to be replaced by

$$
\left(2 \sin \frac{\pi}{k}\right) m \ell=2 n \bar{\ell}-\bar{\ell}^{\prime} .
$$

The solutions we construct will be perturbations of the function $\stackrel{\circ}{U}$ which is the sum of positive copies of $w$ centered at the points $\dot{y}_{j}$, for $j=1, \ldots, m+1$, together with their images under the rotations $R_{k}^{i}=R_{k} \circ \cdots \circ R_{k}$ (i-fold composition), for $i=1, \ldots, k-1$, and copies of $(-1)^{h} w$ (hence with alternating sign) centered at the points $\stackrel{\circ}{z}_{h}$ for $h=$ $1, \ldots, 2 n-1$, together with their images under the rotations $R_{k}^{i}$ for $i=1, \ldots, k-1$. To be more specific, we define

$$
\stackrel{\circ}{U}:=\sum_{i=0}^{k-1}\left(\sum_{j=1}^{m+1} w\left(\cdot-R_{k}^{i} \stackrel{\circ}{y}_{j}\right)+\sum_{h=1}^{2 n-1}(-1)^{h} w\left(\cdot-R_{k}^{i} \stackrel{\circ}{2} h_{h}\right)\right) .
$$

So far, the approximate solution $\stackrel{\circ}{U}$ depends on two discrete parameters (the integers $m$ and $n$ ) and two continuous parameters (the positive reals $\ell$ and $\bar{\ell}$ ) which are related 
by (1.17). It should be clear from the construction that the function $\stackrel{\circ}{U}$ we have constructed is invariant under the action of $D_{k} \times O(N-2)$. Moreover, (1.17) is just a translation of the fact that the length of the rays and the length of the edges of the outer regular polygon are related. The construction of the approximate solution $\stackrel{\circ}{U}$ also depends on the parameter $k$ which defines the dihedral group under the action of which our solution will be invariant. The constraint $k \geq 7$ has a purely geometric origin, roughly speaking, we need $\pi / 2-\pi / k$, which is the angle at $\stackrel{\circ}{m}_{m+1}$ between the edge of the outer regular polygon and the ray joining this vertex to the origin, to be larger than $\pi / 3$ :

$$
\pi / 2-\pi / k>\pi / 3 \text {. }
$$

In turn, this last condition stems from the maximal number of nonoverlapping discs of radius 1 which are tangent to a given disc of radius 1 in the plane. As we will see,

$$
\bar{\ell}=\ell+\mathcal{O}(1) .
$$

Now, let us analyze more carefully the situation at the point $\dot{y}_{m+1}$. When $k \leq 6, \pi / 2-$ $\pi / k<\pi / 3$ and hence the copies of $w$ centered at the points $\stackrel{\circ}{y}_{m}$ and $\stackrel{\circ}{z}_{1}$ interact too much to consider that $\stackrel{\circ}{U}$ is a good approximate solution to our problem, this is just a consequence of the fact that the distance between $\dot{y}_{m}$ and $\stackrel{\circ}{z}_{1}$ can be estimated by

$$
2 \ell \sin \left(\frac{\pi}{4}-\frac{\pi}{2 k}\right)<\ell+\mathcal{O}(1)
$$

when $\ell$ tends to infinity. Therefore, when assuming that $k \geq 7$, we require that the closest neighbors of the point $\stackrel{\circ}{y}_{m}$ be $\stackrel{\circ}{y}_{m+1}, \stackrel{\circ}{z}_{1}$ and $R_{k}^{-1} \stackrel{\circ}{z} 2 n-1_{y^{\prime}}$. Similarly, we require that the closest neighbors of the point $\stackrel{\circ}{z}_{1}$ be $\stackrel{\circ}{y}_{m+1}$ and $\stackrel{\circ}{z}_{2}$.

We now assume that the integer $k \geq 7$ is fixed, and that $m, n$ are two positive integers and $\ell, \bar{\ell}$ are two positive real numbers satisfying (1.17). We further assume that $\ell$ and $\bar{\ell}$ are related by

$$
\Psi(\ell)=\left(2 \sin \frac{\pi}{k}\right) \Psi(\bar{\ell}),
$$

where $\Psi$ is the function defined in (1.11). The origin of this second constraint on the choice of the parameters is not obvious at all. It can be understood either as a balancing condition which is a consequence of a conservation law for solutions of (1.8) (corresponding to the well known balancing formula in the framework of constant mean curvature surfaces), or as a condition which will ensure that the approximate solution we consider is, in a sense to be made precise, very close to a genuine solution of (1.8) (we refer to $\$ 5$ where this second equation will arise and to the Appendix, where some formal justification of this constraint will be given).

Theorem 1.1 is a direct consequence of the following result:

Theorem 1.2. Assume that the integer $k \geq 7$ and the real number $A>0$ are fixed. There exists a positive constant $\ell_{0}>0$ such that, for all $\ell \geq \ell_{0}$, if $\bar{\ell}$ is the solution of

$$
\Psi(\ell)=\left(2 \sin \frac{\pi}{k}\right) \Psi(\bar{\ell}),
$$


if $n, m$ are positive integers satisfying

$$
\left(2 \sin \frac{\pi}{k}\right) m \ell=(2 n-1) \bar{\ell}
$$

and if

$$
m \leq \ell^{A}
$$

then (1.8) has a sign changing solution $u$ which satisfies the symmetry conditions given in (1.15) and (1.16). Moreover

$$
u=\stackrel{\circ}{U}+o(1),
$$

where $o(1) \rightarrow 0$ uniformly in $\mathbb{R}^{N}$ as $\ell \rightarrow \infty$, and the energy of $u$ is finite and can be expanded as

$$
\mathcal{E}(u)=k(m+2 n) \mathcal{E}(w)+o(1)
$$

where $o(1) \rightarrow 0$ as $\ell \rightarrow \infty$.

Remark 1.2. The condition $m \leq \ell^{A}$ is purely technical and is a drawback of our proof. In fact, by going carefully through the last arguments of the proof, it is clear that this condition can already be weakened to handle the cases where

$$
m \leq e^{A \ell}
$$

for some fixed $A>0$, chosen small enough. What is more, we are convinced that this condition can be completely removed by choosing different weighted norms on the spaces of functions we are considering (see [8] and [9]). Since this would enlarge the size of the paper considerably, we have chosen not to follow this route.

Observe that, once $\ell$ is fixed large enough, the constant $\bar{\ell}$ is given uniquely by (1.25). Therefore, the existence of solutions of (1.8) depends on our ability to solve (1.26) for some integers $m, n$. Indeed, it follows from (1.25) that $\bar{\ell}$ is implicitly given as a function of $\ell$ (provided the latter is large enough) and that it can be expanded, in powers of $\ell$, as

$$
\bar{\ell}=\ell+\ln \left(2 \sin \frac{\pi}{k}\right)+\mathcal{O}\left(\ell^{-1}\right)
$$

as $\ell$ tends to $\infty$. Inserting this information back into (1.26), we find using Lemma 5.1, that (1.26) reduces to

$$
\frac{2 n-1}{m}=2 \sin \frac{\pi}{k}\left(1-\ln \left(2 \sin \frac{\pi}{k}\right) \ell^{-1}+\mathcal{O}\left(\ell^{-2}\right)\right) .
$$

We are now in a position to give examples of such solutions. Certainly, for any integer $m \geq 1$, one can choose $n \in \mathbb{N}$ such that

$$
1 \leq 2 n-1-\left(2 \sin \frac{\pi}{k}\right) m<3 .
$$


Then, provided $m$ is chosen large enough, there will exist a unique $\ell>\ell_{0}$ satisfying (1.26), and (1.31) together with (1.30) implies that there exist positive constants $C_{1}<C_{2}$ such that

$$
C_{1} m \leq \ell \leq C_{2} m
$$

Theorem 1.2 then ensures the existence of solutions of (1.8) for each such choice of the integer $m$.

To complete the description of our construction, let us briefly comment on the relation between this result and the corresponding construction for constant mean curvature surfaces in Euclidean 3-space. As already mentioned, the construction in the present paper follows very closely a similar construction of compact constant mean curvature surfaces given in [9]. In that framework one tries to construct compact constant mean curvature surfaces in Euclidean 3-space by connecting together spheres of radius 1 which are tangent. In the initial configuration, the centers of the spheres can be arranged along the edges of a very large regular polygon and also along the rays joining the center to the vertices of the polygon. It is proven in [9] that a perturbation argument can be applied and, as a result, a compact constant mean curvature surface is obtained (provided the size of the polygon is large enough). This surface can be constructed in such a way that the pieces which are close to the rays joining the origin to the vertices are embedded and close to embedded constant mean curvature surfaces which are known as unduloids, while the pieces which are close to the edges of the regular polygon are immersed constant mean surfaces which are close to nodoids (in our framework, this corresponds to the fact that we arrange solutions with the same sign along the rays joining the origin to the vertices of the polygon and solutions with alternating sign along the edges of the polygon). A similar construction has also been obtained by Jleli and Pacard in [8].

Remark 1.3. For the sake of simplicity, we have chosen to present the proof of the existence of solutions which are invariant under the action of a rather large symmetry group. However, a more general construction (i.e. leading to solutions of (1.8) having less symmetry) can be obtained as in the case of constant mean curvature surfaces [9]; we shall address this problem in a forthcoming paper.

In the next section, we describe more carefully the solution predicted in the above theorem and we give an overview of the proof and the plan of the paper.

\section{Ansatz and sketch of the proof}

We construct a finite-dimensional family of approximate solutions of (1.8) which are close to $\stackrel{\circ}{U}$ and depend on $2 n+m$ parameters which we now define. These approximate solutions are in fact equal to $\stackrel{\circ}{U}$ when all the parameters are set to 0 . This time, instead of centering the copies of $\pm w$ at the points $\stackrel{\circ}{y}_{j}, \stackrel{\circ}{z}_{h}$ as well as at their images under the rotations $R_{k}^{i}$ for $i=1, \ldots, k-1$, we center the copies of $\pm w$ at points which are small perturbations of the points $\stackrel{\circ}{y}_{j}, \stackrel{\circ}{z}_{h}$. To make this precise, we define

$$
y_{j}:=\stackrel{\circ}{y}_{j}+\alpha_{j} e_{1} \quad \text { for } j=1, \ldots, m+1,
$$


and

$$
z_{h}:=\stackrel{\circ}{z}_{h}+\beta_{h} \mathrm{t}+\bar{\ell} \gamma_{h} \mathrm{n}, \quad \text { for } \quad h=1, \ldots, 2 n-1,
$$

where $\bar{\ell}$ has been defined in (1.24), t has been defined in (1.21), and

$$
\mathrm{n}:=\cos \frac{\pi}{k} \mathrm{e}_{1}+\sin \frac{\pi}{k} \mathrm{e}_{2} .
$$

Observe that, since we assume that our construction is invariant under the dihedral group generated by $\Gamma$ and $R_{k}$ (see (1.16)), the points $z_{h}$ and $z_{2 n-h}$ are related by

$$
z_{2 n-h}=R_{k}\left(\Gamma z_{h}\right) \quad \text { for all } h=1, \ldots, n .
$$

In other words, if we rotate by $R_{k}$ the point obtained by reflecting $z_{h}$ with respect to the plane $x_{2}=0$ we get $z_{2 n-h}$. Since $R_{k}(\Gamma \mathrm{t})=-\mathrm{t}$ and $R_{k}(\Gamma \mathrm{n})=\mathrm{n}$, this implies that we necessarily have

$$
\beta_{h}=-\beta_{2 n-h} \quad \text { and } \quad \gamma_{h}=\gamma_{2 n-h} \quad \text { for } h=1, \ldots, n
$$

and in particular $\beta_{n}=0$. We thus conclude that there are only $2 n+m$ free parameters.

We will assume that the parameters which appear in the definition of both $y_{j}$ and $z_{h}$ satisfy

$$
\begin{aligned}
\left|\alpha_{j}\right| \leq 1 & \text { for } j=1, \ldots, m+1, \\
\left|\beta_{h}\right| \leq 1 & \text { for } h=1, \ldots, n-1, \\
\ell\left|\gamma_{h}\right| \leq 1 & \text { for } h=1, \ldots, n .
\end{aligned}
$$

In these inequalities, the bound 1 can be replaced by any positive constant.

The set of points where the copies of $w$ will be centered is now given by

$$
\Pi:=\bigcup_{i=0}^{k-1}\left(\left\{R_{k}^{i} y_{j}: j=1, \ldots, m+1\right\} \cup\left\{R_{k}^{i} z_{h}: h=1, \ldots, 2 n-1\right\}\right),
$$

and we now look for a solution of (1.8) of the form $u=U+\phi$, where

$$
U(x):=\sum_{i=0}^{k-1}\left(\sum_{j=1}^{m+1} w\left(x-R_{k}^{i} y_{j}\right)+\sum_{h=1}^{2 n-1}(-1)^{h} w\left(x-R_{k}^{i} z_{h}\right)\right) .
$$

Observe that, by construction, the function $U$ satisfies the symmetry assumptions (1.15) and (1.16). We define

$$
\begin{aligned}
L & :=\Delta-1+p|U|^{p-1}, \\
E & :=|U|^{p-1} U-\sum_{i=0}^{k-1}\left(\sum_{j=1}^{m+1} w^{p}\left(\cdot-R_{k}^{i} y_{j}\right)+\sum_{h=1}^{2 n-1}(-1)^{h} w^{p}\left(\cdot-R_{k}^{i} z_{h}\right)\right), \\
Q(\phi) & :=|U+\phi|^{p-1}(U+\phi)-|U|^{p-1} U-p|U|^{p-1} \phi .
\end{aligned}
$$

Observe that both $E$ and $Q$ depend implicitly on the parameters $\alpha_{j}, \beta_{h}$ and $\gamma_{h}$ even though this is not apparent in the notations. With these notations, the solvability of (1.8) reduces 
to finding parameters $\alpha_{j}, \beta_{h}$ and $\gamma_{h}$ and a function $\phi$ which are solutions of the nonlinear problem

$$
L \phi+E+Q(\phi)=0 \quad \text { in } \mathbb{R}^{N}
$$

such that $\phi$ tends to 0 as $|x|$ tends to $\infty$.

Remark 2.1. We will solve (2.10) in the class of functions $\phi$ satisfying (1.15) and (1.16). Therefore, from now on, we always assume that all the functions we consider satisfy (1.15) and (1.16) without further mention.

In order to solve this highly nonlinear problem, we apply a Lyapunov-Schmidt type reduction argument: first, we solve a projected problem which allows us to reduce the solvability of (2.10) to the solvability of some finite-dimensional nonlinear system (called the reduced problem); then, in a second step, we will explain how to solve this reduced problem.

To proceed, we assume that real numbers $\ell, \bar{\ell}$ are chosen so that (1.24) holds and that integers $n$ and $m$ satisfy (1.17). We consider a cut-off function $\chi \in \mathcal{C}^{\infty}(\mathbb{R})$ such that

$$
\chi(s) \equiv 1 \quad \text { for } s \leq-1, \quad \chi(s) \equiv 0 \quad \text { for } s \geq 0 .
$$

We fix a constant $\zeta>0$ (independent of $\ell$ and the choice of the parameters $\alpha_{j}, \beta_{h}$ and $\gamma_{h}$ satisfying the constraints (2.4)) so that the balls of radius $(\ell-\zeta) / 2$ centered at different points of $\Pi$ are mutually disjoint for all $\ell$ large enough. This is possible thanks to (1.29) and our geometric assumption (namely $k \geq 7$ ) which implies that the minimum distance between two different points of $\Pi$ is bounded from below by $\ell-\zeta_{0}$ for some constant $\zeta_{0}>0$ independent of $\ell$ large enough (say $\ell \geq \ell_{0}$ ). Observe that when $k \leq 6$ the distance between $y_{m}$ and $z_{1}$ can be estimated by $2 \sin \left(\frac{\pi}{4}-\frac{\pi}{2 k}\right) \ell+\mathcal{O}(1)$ as $\ell$ tends to $\infty$, and therefore a proper choice of $\zeta$ would not be possible in this case since $2 \sin \left(\frac{\pi}{4}-\frac{\pi}{2 k}\right)<1$. We define the compactly supported vector field

$$
\Xi(x):=\chi(2|x|-\ell+\zeta) \nabla w(x) .
$$

Observe that, by construction (in fact given the choice of $\zeta$ ), we have, for all $y, z \in \Pi$,

$$
\int_{\mathbb{R}^{N}}\left(\mathrm{e}_{i} \cdot \Xi(\cdot-y)\right)\left(\mathrm{e}_{j} \cdot \Xi(\cdot-z)\right) d x=0 \quad \text { if } i \neq j \text { or } y \neq z .
$$

It will be convenient to define the function

$$
M(c, d):=\sum_{i=0}^{k-1}\left(\sum_{j=1}^{m+1} R_{k}^{i} c_{j} \cdot \Xi\left(\cdot-R_{k}^{i} y_{j}\right)+\sum_{h=1}^{2 n-1} R_{k}^{i} d_{h} \cdot \Xi\left(\cdot-R_{k}^{i} z_{h}\right)\right),
$$

as well as the operator

$$
\mathcal{L}(\phi, c, d):=L \phi+M(c, d),
$$

where $\phi$ is a function defined on $\mathbb{R}^{N}$, the $(m+1)$-tuple

$$
c:=\left(c_{1}, \ldots, c_{m+1}\right) \in\left(\mathbb{R} e_{1}\right)^{m+1},
$$


and the $(2 n-1)$-tuple

$$
d:=\left(d_{1}, \ldots, d_{2 n-1}\right) \in\left(\mathbb{R} e_{1} \oplus \mathbb{R} e_{2}\right)^{2 n-1} .
$$

Observe that, given the symmetries we impose on all the functions we deal with (see Remark 2.1), the function $M(c, d)$ has to be invariant under the action of both $R_{k}$ and $\Gamma$, and this implies that, for $h=1, \ldots, n$, the vectors $d_{h}$ and $d_{2 n-h}$ are related by

$$
d_{2 n-h}=R_{k}\left(\Gamma d_{h}\right)
$$

and hence $d_{2 n-h}$ can be expressed in terms of $d_{h}$ as

$$
d_{2 n-h}=-\left(d_{h} \cdot \mathrm{t}\right) \mathrm{t}+\left(d_{h} \cdot \mathrm{n}\right) \mathrm{n} .
$$

In the next section, we will define suitable function spaces in which the equation

$$
\mathcal{L}(\phi, c, d)=g \quad \text { in } \mathbb{R}^{N}
$$

admits a solution which tends to 0 as $|x|$ tends to $\infty$ and which satisfies the orthogonality conditions

$$
\begin{array}{ll}
\int_{\mathbb{R}^{N}} \phi e_{1} \cdot \Xi\left(\cdot-y_{j}\right) d x=0 & \text { for } j=1, \ldots, m+1, \\
\int_{\mathbb{R}^{N}} \phi e_{i} \cdot \Xi\left(\cdot-z_{h}\right) d x=0 & \text { for } h=1, \ldots, n \text { and } i=1,2 .
\end{array}
$$

Again, given the symmetries we impose (see Remark 2.1), a function $\phi$ satisfies (2.17) and (2.18) if and only if

$$
\int_{\mathbb{R}^{N}} \phi e_{i} \cdot \Xi(\cdot-y) d x=0 \quad \text { for all } i=1, \ldots, N \text { and } y \in \Pi .
$$

In order to study the operator $\mathcal{L}$, the key idea is that the linear operator $L$ is close to being the sum of many copies of

$$
L_{0}=\Delta-1+p w^{p-1}
$$

centered at the points of $\Pi$, and we take advantage of the fact that the invertibility of $L_{0}$ is well understood.

Once the linear theory is understood, we will consider the following nonlinear projected problem: given the points $y_{j}$ and $z_{h}$ defined in (2.1), (2.2) and satisfying (2.4), find a function $\phi$ satisfying the symmetry assumptions (1.15), (1.16), the orthogonality conditions (2.17), (2.18) and tending to 0 as $|x|$ tends to $\infty$, and find vectors $c_{j}, d_{h}$ such that

$$
\mathcal{L}(\phi, c, d)+E+Q(\phi)=0 \quad \text { in } \mathbb{R}^{N} .
$$

In the next sections, we show unique solvability of (2.20) by means of a fixed point argument and we prove that the solution $\phi$ depends continuously (in fact, with more work one can prove that it depends smoothly) on the points $y_{j}$ and $z_{h}$. To achieve this, we first study the solvability of a linear problem and then apply some standard fixed point theorem for contraction mappings to solve the nonlinear problem. 


\section{Linear theory}

The main result of this section is concerned with the solvability of (2.16), uniformly in $\ell$, as $\ell$ tends to $\infty$, and also uniformly in the parameters $\alpha_{j}, \beta_{h}$ and $\gamma_{h}$ satisfying the constraints (2.4). We henceforth assume that the real numbers $\ell, \bar{\ell}$ are chosen so that (1.24) holds and that the integers $n$ and $m$ satisfy (1.17). In particular,

$$
\bar{\ell}=\ell+\mathcal{O}(1) .
$$

We prove that, provided $\ell$ is large enough, the linear operator $\mathcal{L}$ defined in the previous section in (2.15) has nice mapping properties.

Given $\eta<0$, we consider the weighted norm

$$
\|g\|_{*}:=\sup _{x \in \mathbb{R}^{N}}\left|\left(\sum_{y \in \Pi} e^{\eta|x-y|}\right)^{-1} g(x)\right|,
$$

where we recall that the set $\Pi$ was defined in (2.5).

With this definition at hand, we prove the following a priori estimate:

Lemma 3.1. Assume that $\eta<0$ is fixed. There exist $\ell_{0}, \delta_{0}, C>0$ (all depending on the choice of $\eta$ ) such that, for all $\ell>\ell_{0}$,

$$
\sup _{i=1, \ldots, m+1}\left|c_{i}\right|+\sup _{h=1, \ldots, n}\left|d_{h}\right| \leq C\left(\|\mathcal{L}(\phi, c, d)\|_{*}+e^{-\delta_{0} \ell}\|\phi\|_{*}\right) .
$$

Observe that the estimate does not depend on $n$ or $m$, nor on $\ell$ provided the latter is chosen large enough. Further observe that

$$
\sup _{h=1, \ldots, n}\left|d_{h}\right|=\sup _{h=1, \ldots, 2 n-1}\left|d_{h}\right|,
$$

since $d_{2 n-h}=d_{h}$ for $h=1, \ldots, n$.

Proof. Let us give a detailed proof of the estimate for the coefficient $c_{1}$. We start with the definition of $\mathcal{L}$ given in (2.15) which we multiply by $e_{1} \cdot \Xi\left(\cdot-y_{1}\right)$. Using some integration by parts together with (2.13), we obtain

$$
\begin{aligned}
c_{1} \cdot \mathrm{e}_{1} \int_{\mathbb{R}^{N}}\left(\mathrm{e}_{1} \cdot \Xi\left(\cdot-y_{1}\right)\right)^{2} d x= & \int_{\mathbb{R}^{N}} \mathcal{L}(\phi, c, d)\left(\mathrm{e}_{1} \cdot \Xi\left(\cdot-y_{1}\right)\right) d x \\
& -\int_{\mathbb{R}^{N}} \phi L\left(\mathrm{e}_{1} \cdot \Xi\left(\cdot-y_{1}\right)\right) d x .
\end{aligned}
$$

Obviously,

$$
\lim _{\ell \rightarrow \infty} \int_{\mathbb{R}^{N}}\left(e_{1} \cdot \Xi\left(\cdot-y_{1}\right)\right)^{2} d x=\frac{1}{N} \int_{\mathbb{R}^{N}}|\nabla w|^{2} d x
$$

therefore

$$
\int_{\mathbb{R}^{N}}\left(\mathrm{e}_{1} \cdot \Xi\left(\cdot-y_{1}\right)\right)^{2} d x \geq C_{0}>0
$$


for all $\ell$ large enough (say $\ell>\ell_{0}$ ). Thanks to (1.10), we know that $|\Xi|$ is bounded and hence we can estimate

$$
\left|\int_{\mathbb{R}^{N}} \mathcal{L}(\phi, c, d)\left(\mathrm{e}_{1} \cdot \Xi\left(\cdot-y_{1}\right)\right) d x\right| \leq C\|\mathcal{L}(\phi, c, d)\|_{*}
$$

provided $\ell>\ell_{0}$. Finally, since

$$
L_{0}\left(\mathrm{e}_{1} \cdot \nabla w\right)=\left(\Delta-1+p w^{p-1}\right)\left(\mathrm{e}_{1} \cdot \nabla w\right)=0,
$$

we can write

$$
L\left(e_{1} \cdot \Xi\left(\cdot-y_{1}\right)\right)=L\left(e_{1} \cdot \Xi\left(\cdot-y_{1}\right)\right)-L_{0}\left(e_{1} \cdot \nabla w\left(\cdot-y_{1}\right)\right)
$$

and, using this, it is easy to check that there exist constants $C, \delta_{0}>0$ such that

$$
\left|\int_{\mathbb{R}^{N}} \phi L\left(\mathrm{e}_{1} \cdot \Xi\left(\cdot-y_{1}\right)\right) d x\right| \leq C e^{-\delta_{0} \ell}\|\phi\|_{*},
$$

where $\delta_{0}>0$ depends on $\eta<0$. To obtain the last estimate, two different effects have to be taken into account: the first one is the effect of the Laplace operator on the cut-off function $\chi$ which is used to define $\Xi$ and the second one is the difference between the two potentials $p|U|^{p-1}$ and $p w^{p-1}$ which appear respectively in the definition of $L$ and $L_{0}$. The proof of the estimate for $c_{1}$ follows at once from the above estimates. A similar proof holds for the estimates for any $c_{j}$ and any $d_{h}$.

Thanks to the previous estimate, we can prove the following:

Proposition 3.1. Assume that $\eta \in(-1,0)$ is fixed. There exist $\ell_{0}, C>0$ such that, for all $\ell>\ell_{0}$,

$$
\|\phi\|_{*} \leq C\|\mathcal{L}(\phi, c, d)\|_{*}
$$

provided $\phi$ satisfies (2.17) and (2.18).

Again, it is worth mentioning that the estimate does not depend on $n, m$, nor on $\ell$ provided the latter is chosen large enough.

Proof. Using the definition of the function $M(c, d)$ given in (2.14), we have

$$
\|M(c, d)\|_{*} \leq C\left(\sup _{i=1, \ldots, m+1}\left|c_{i}\right|+\sup _{h=1, \ldots, n}\left|d_{h}\right|\right) .
$$

The above inequality follows from the observation that, by definition, the vector field $\Xi$ decays exponentially, more precisely, there exists a constant $C>0$ such that $|\Xi| \leq$ $C e^{-|x|}$ in $\mathbb{R}^{N}$. Since we assume that $\eta \in(-1,0)$, a direct consequence of the previous lemma is that

$$
\|M(c, d)\|_{*} \leq C\left(\sup _{i=1, \ldots, m+1}\left|c_{i}\right|+\sup _{h=1, \ldots, n}\left|d_{h}\right|\right) \leq C\left(\|\mathcal{L}(\phi, c, d)\|_{*}+e^{-\delta_{0} \ell}\|\phi\|_{*}\right)
$$

for some constant $C>0$ which does not depend on $\ell>\ell_{0}$. 
It is easy to check that the function

$$
W:=\sum_{y \in \Pi} e^{\eta|\cdot-y|}
$$

satisfies

$$
L W \leq-\frac{1-\eta^{2}}{2} W
$$

in $\mathbb{R}^{N} \backslash \bigcup_{y \in \Pi} B(y, \rho)$ provided $\rho$ is fixed large enough (independently of $\ell \geq \ell_{0}$ ). Indeed, for all $y \in \Pi$, we can write

$$
L e^{\eta|x-y|}=-\left(1-\eta^{2}-\frac{N-1}{|x-y|} \eta-p|U|^{p-1}\right) e^{\eta|x-y|} \leq-\frac{1-\eta^{2}}{2} e^{\eta|x-y|}
$$

provided $\operatorname{dist}(x, \Pi)$ is large enough, since $|U|^{p-1}$ tends to 0 away from the points of $\Pi$.

Making use of the fact that $\eta \in(-1,0)$ together with the maximum principle, we conclude that the function $W$ can be used as a barrier to prove the pointwise estimate

$$
|\phi|(x) \leq C\left(\|L \phi\|_{*}+\sup _{y \in \Pi}\|\phi\|_{L^{\infty}(\partial B(y, \rho))}\right) W(x)
$$

for all $x \in \mathbb{R}^{N} \backslash \bigcup_{y \in \Pi} B(y, \rho)$.

Granted this preliminary estimate, the proof goes by contradiction. Let us assume there exist a sequence of $\ell$ tending to $\infty$ and a sequence of solutions of (2.16) for which the inequality (3.2) is not true. The problem being linear, we can reduce to the case where we have a sequence $\ell^{(i)}$ tending to $\infty$ and sequences $\phi^{(i)}, c^{(i)}, d^{(i)}$ such that

$$
\left\|\mathcal{L}\left(\phi^{(i)}, c^{(i)}, d^{(i)}\right)\right\|_{*} \rightarrow 0 \text { and }\left\|\phi^{(i)}\right\|_{*}=1 .
$$

But (3.3) implies that we also have $\left\|M\left(c^{(i)}, d^{(i)}\right)\right\|_{*} \rightarrow 0$ and hence $\left\|L \phi^{(i)}\right\|_{*} \rightarrow 0$. Then, (3.4) implies that there exists $y^{(i)} \in \Pi$ such that

$$
\left\|\phi^{(i)}\right\|_{L^{\infty}\left(B\left(y^{(i)}, \rho\right)\right)} \geq C
$$

for some fixed constant $C>0$. Using elliptic estimates together with Ascoli-Arzelà's theorem, we can extract from $\phi^{(i)}\left(\cdot-y^{(i)}\right)$ a subsequence which converges (uniformly on compact sets) to $\phi_{\infty}$ which is a solution of

$$
\left(\Delta-1+p w^{p-1}\right) \phi_{\infty}=0 \quad \text { in } \mathbb{R}^{N}
$$

and which is bounded by a constant times $e^{\eta|x|}$, with $\eta<0$. Moreover, recall that $\phi^{(i)}$ satisfies the orthogonality conditions (2.17) and (2.18) and also satisfies some symmetry properties which are described in Remark 2.1 (and in particular, this implies that $\phi^{(i)}$ satisfies (2.19)). Using this and passing to the limit, one checks that the limit function $\phi_{\infty}$ also satisfies

$$
\int_{\mathbb{R}^{N}} \phi_{\infty} \partial_{x_{j}} w d x=0 \quad \text { for } j=1, \ldots, N
$$

But the solution $w$ being nondegenerate, this implies that $\phi_{\infty} \equiv 0$, which is certainly in contradiction with (3.5). 
We are now in a position to prove the main result of this section:

Proposition 3.2. Assume that $\eta \in(-1,0)$ is fixed. There exist $\ell_{0}, C>0$ such that, for all $\ell>\ell_{0}$, and for all $g \in L^{\infty}\left(\mathbb{R}^{N}\right)$ satisfying $\|g\|_{*}<\infty$, there exists a unique triple $(\phi, c, d)$ such that

$$
\mathcal{L}(\phi, c, d)=g \quad \text { in } \mathbb{R}^{N}
$$

and $\phi$ satisfies (2.17) and (2.18). Moreover

$$
\sup _{i=1, \ldots, m+1}\left|c_{i}\right|+\sup _{h=1, \ldots, h}\left|d_{h}\right|+\|\phi\|_{*} \leq C\|g\|_{*} .
$$

As in the previous results, it is important to notice that the estimate does not depend on the integers $n, m$, nor on $\ell$ provided the latter is chosen large enough.

Proof. We consider the Hilbert space

$$
\mathcal{H}=\left\{\phi \in H^{1}\left(\mathbb{R}^{N}\right): \int_{\mathbb{R}^{N}} \phi e \cdot \Xi(\cdot-y) d x=0, \forall y \in \Pi, \forall e \in \mathbb{R}^{N},|e|=1\right\},
$$

and, as usual, we also assume that the functions enjoy the symmetries described in Remark 2.1.

Assume that we are given $g \in L^{2}\left(\mathbb{R}^{N}\right)$. Standard arguments (i.e. Lax-Milgram's Theorem) imply that

$$
\phi \in \mathcal{H} \mapsto \frac{1}{2} \int_{\mathbb{R}^{N}}\left(|\nabla \phi|^{2}+\phi^{2}\right) d x+\int_{\mathbb{R}^{N}} \phi g d x
$$

has a unique minimizer $\phi \in \mathcal{H}$ (here we implicitly use the fact that $\eta<0$ so that the last term is a continuous linear functional defined in $\mathcal{H}$ ). Then $\phi$ is the unique weak solution of

$$
\begin{aligned}
\Delta \phi-\phi-g \in & \operatorname{Span}\left\{\sum_{i=0}^{k-1} R_{k}^{i} e_{1} \cdot \Xi\left(\cdot-R_{k}^{i} y_{j}\right): j=1, \ldots, m+1\right\} \\
& \oplus \operatorname{Span}\left\{\sum_{i=0}^{k-1} R_{k}^{i} e_{j} \cdot \Xi\left(\cdot-R_{k}^{i} z_{h}\right): j=1,2, h=1, \ldots, n\right\},
\end{aligned}
$$

which belongs to $\mathcal{H}$. In other words, if we define the operator

$$
\mathcal{L}_{0}(\phi, c, d):=\Delta \phi-\phi+M(c, d),
$$

we have uniquely solved

$$
\mathcal{L}_{0}(\phi, c, d)=g
$$

for $\phi \in \mathcal{H}, c_{j} \in \mathbb{R} e_{1}$ and $d_{h} \in \mathbb{R} e_{1} \oplus \mathbb{R} e_{2}$. Thanks to the above arguments

$$
\mathcal{L}_{0}^{-1}: L^{2}\left(\mathbb{R}^{N}\right) \rightarrow \mathcal{H} \times\left(\mathbb{R} e_{1}\right)^{m+1} \times\left(\mathbb{R} e_{1} \oplus \mathbb{R} e_{2}\right)^{2 n-1}
$$

is well defined. 
The solvability of

$$
\mathcal{L}(\phi, c, d)=g
$$

in $\mathcal{H} \times\left(\mathbb{R} e_{1}\right)^{m+1} \times\left(\mathbb{R} e_{1} \oplus \mathbb{R} e_{2}\right)^{2 n-1}$ can then by rephrased as the invertibility of the operator $I+\mathcal{K}$, where

$$
\mathcal{K}(\phi, c, d):=\mathcal{L}_{0}^{-1}\left(p|U|^{p-1} \phi\right) .
$$

Using the fact that $U$ decays exponentially at $\infty$, it is easy to check that the operator $\mathcal{K}$ is compact, hence the invertibility of (3.7) follows from Fredholm theory. Injectivity follows from Proposition 3.1 and Lemma 3.1. The Fredholm alternative implies that $I+\mathcal{K}$ is therefore an isomorphism provided $\ell$ is chosen large enough.

So far, we have obtained a function $\phi$ solving $L \phi+M(c, d)=g$ which belongs to $H^{1}\left(\mathbb{R}^{N}\right)$, but elliptic regularity implies that $\phi \in L^{\infty}\left(\mathbb{R}^{N}\right)$. This completes the proof of the existence of the solution. The uniqueness and the corresponding estimate follow at once from Proposition 3.1 and Lemma 3.1.

\section{The nonlinear projected problem}

We keep the notation and assumptions of the previous sections. In this section, we prove that we can apply some fixed point theorem for contraction mappings to solve the nonlinear problem

$$
\mathcal{L}(\phi, c, d)+E+Q(\phi)=0 \quad \text { in } \mathbb{R}^{N}
$$

provided the parameter $\ell$ is chosen large enough. This is the content of the following:

Proposition 4.1. Assume that $\eta \in(-1,0)$ is fixed. Then there exist $\ell_{0}, C>0$ such that, for all $\ell \geq \ell_{0}$, there exists a solution $(\phi, c, d)$ to problem (4.1) such that $\phi$ satisfies (2.17) and (2.18). This solution depends continuously on the parameters of the construction (namely $\alpha_{j}, \beta_{h}$ and $\gamma_{h}$ ) and satisfies

$$
\sup _{i=1, \ldots, m+1}\left|c_{i}\right|+\sup _{h=1, \ldots, n}\left|d_{h}\right|+\|\phi\|_{*} \leq C e^{-\delta_{1} \ell},
$$

where $\delta_{1}=\min (1,(p+\eta) / 2)$.

Before we proceed with the proof of this result, let us briefly comment on the value of the constant $\delta_{1}$. Observe that given $p>1$ it is always possible to choose $\eta \in(-1,0)$ such that

$$
p+\eta>1,
$$

and hence $\delta_{1}>1 / 2$ for this choice. We shall assume from now on that $\eta$ is chosen so that $\delta_{1}>1 / 2$.

Proof. The proof relies on a classical fixed point argument for contraction mappings together with the estimates we now derive. The constants $\left(C_{j}\right.$ or $\left.C\right)$ below do not depend on $\ell$ provided $\ell$ is chosen large enough. First of all, there exists $C_{0}>0$ such that

$$
\|E\|_{*} \leq C_{0}\left(e^{-\ell}+e^{-(p+\eta) \ell / 2}\right) .
$$


In fact, let us assume that we want to estimate $E$ near $y_{1}$. In the ball of radius $\ell / 2$ centered at $y_{1}$, one can use the fact that

$$
U=w\left(\cdot-y_{1}\right)+\mathcal{O}\left(\ell^{(1-N) / 2} e^{\left|--y_{1}\right|-\ell}\right)
$$

to expand $E$ as

$$
\begin{aligned}
|E| & =\left|\left(w\left(\cdot-y_{1}\right)+\mathcal{O}\left(\ell^{(1-N) / 2} e^{\left|--y_{1}\right|-\ell}\right)\right)^{p}-w^{p}\left(\cdot-y_{1}\right)+\mathcal{O}\left(\ell^{p(1-N) / 2} e^{p\left(\left|-y_{1}\right|-\ell\right)}\right)\right| \\
& \leq C e^{(2-p)\left|\cdot-y_{1}\right|-\ell} \\
& \leq C\left(e^{-\ell}+e^{-(p+\eta) \ell / 2}\right) e^{\eta\left|\cdot-y_{1}\right|} \\
& \leq C\left(e^{-\ell}+e^{-(p+\eta) \ell / 2}\right) \sum_{y \in \Pi} e^{\eta|\cdot-y|} .
\end{aligned}
$$

This can be done for any point of $\Pi$. Away from the balls of radius $\ell / 2$ centered at the points of $\Pi$, we take advantage of the fact that $U$ decays exponentially to prove that

$$
|E| \leq C \sum_{y \in \Pi} e^{-p|\cdot-y|} \leq C e^{-(p+\eta) \ell / 2} \sum_{y \in \Pi} e^{\eta|\cdot-y|} .
$$

The estimate for $E$ then follows at once.

We choose $\delta_{1}=\min (1,(p+\eta) / 2)$ and we set

$$
C_{1}:=\frac{4 C_{0}}{\left\|\mathcal{L}^{-1}\right\|}
$$

where $\mathcal{L}^{-1}$ is the inverse of $\mathcal{L}$, which has been proved to exist in Proposition 3.2. Taylor's expansion implies that there exist $\delta_{2}, C_{2}>0$ such that

$$
\left\|Q\left(\phi_{1}\right)-Q\left(\phi_{2}\right)\right\|_{*} \leq C_{2} e^{-\delta_{2} \ell}\left\|\phi_{1}-\phi_{2}\right\|_{*}
$$

for all $\phi_{1}, \phi_{2}$ satisfying $\left\|\phi_{j}\right\|_{*} \leq C_{1} e^{-\delta_{1} \ell}$. Some care is needed to derive the last estimate. Essentially, the estimate follows from the observation that at a point where $U \neq 0$ and $\left|\phi_{1}\right|+\left|\phi_{2}\right| \leq|U| / 2$, one can use the inequality

$$
\left|Q\left(\phi_{2}\right)-Q\left(\phi_{1}\right)\right| \leq C|U|^{p-2}\left(\left|\phi_{1}\right|+\left|\phi_{2}\right|\right)\left|\phi_{2}-\phi_{1}\right|,
$$

while at a point where $\left|\phi_{1}\right|+\left|\phi_{1}\right| \geq|U| / 2$, one can use

$$
\left|Q\left(\phi_{2}\right)-Q\left(\phi_{1}\right)\right| \leq C\left(\left|\phi_{1}\right|^{p-1}+\left|\phi_{2}\right|^{p-1}\right)\left|\phi_{2}-\phi_{1}\right| .
$$

Proposition 3.2 allows one to rewrite (4.1) as a fixed point problem

$$
(\phi, c, d)=-\mathcal{L}^{-1}(E+Q(\phi)) .
$$

Provided $\ell$ is chosen large enough, the above estimates readily yield the existence of a unique fixed point in the ball of radius $C_{1} e^{-\delta_{1} \ell}$ in the space $L_{*}^{\infty}\left(\mathbb{R}^{N}\right) \times\left(\mathbb{R} e_{1}\right)^{m+1} \times$ $\left(\mathbb{R} e_{1} \oplus \mathbb{R} e_{2}\right)^{2 n-1}$, where

$$
L_{*}^{\infty}\left(\mathbb{R}^{N}\right):=\left\{\phi \in L^{\infty}\left(\mathbb{R}^{N}\right):\|\phi\|_{*}<\infty\right\},
$$


which is endowed with the norm $\|\cdot\|_{*}$, and $\left(\mathbb{R} e_{1}\right)^{m+1}$ and $\left(\mathbb{R} e_{1} \oplus \mathbb{R} e_{2}\right)^{2 n-1}$ are endowed with the natural norms, namely

$$
\|c\|:=\sup _{j=1, \ldots, m+1}\left|c_{j}\right|, \quad\|d\|:=\sup _{h=1, \ldots, n}\left|d_{h}\right|=\sup _{h=1, \ldots, 2 n-1}\left|d_{h}\right| .
$$

This completes the proof of the existence of a solution of (4.1).

Observe that elliptic estimates imply that the solution we have obtained also satisfies

$$
\|\phi\|_{*}+\|\nabla \phi\|_{*}+\left\|\nabla^{2} \phi\right\|_{*} \leq C_{3} e^{-\delta_{1} \ell}
$$

for some constant $C_{3}>0$.

It remains to check that the solution we have obtained depends continuously on the parameters of our construction, $\alpha_{j}, \delta_{h}$ and $\gamma_{h}$. Usually, verifying this property is standard, but here some care is needed since the dependence of the nonlinear operator on the parameters is quite intricate and not explicit. Indeed, the parameters appear in the definition of $\mathcal{L}$ and also in the definition of the function space $\mathcal{H}$ and hence they implicitly appear in the construction of $\mathcal{L}^{-1}$.

Now, assume that we have two solutions corresponding to two sets of parameters. Say

$$
L \phi+M(c, d)+E+Q(\phi)=0
$$

corresponding to the points $y_{j}$ and $z_{h}$, and

$$
\dot{L} \dot{\phi}+\dot{M}(\dot{c}, \dot{d})+\dot{E}+\dot{Q}(\dot{\phi})=0
$$

corresponding to the points $\dot{y}_{j}$ and $\dot{z}_{h}$ (we will adorn all functions and operators with a when they correspond to the points $\dot{y}_{j}$ and $\dot{z}_{h}$ ). Observe that, by construction, $\dot{\phi}$ is $L^{2}$ orthogonal to $e_{j} \cdot \dot{\Xi}$ while $\phi$ is $L^{2}$-orthogonal to $e_{j} \cdot \Xi$. First, we choose $\gamma$ and $\delta$ so that

$$
\dot{\bar{\phi}}:=\dot{\phi}-M(\gamma, \delta)
$$

satisfies the same orthogonality condition as $\phi$ (namely, is $L^{2}$-orthogonal to $\Xi$ ). Then, we rewrite the equation satisfied by $\dot{\phi}$ as

$$
L \dot{\bar{\phi}}+M(\dot{c}, \dot{d})+(\dot{L}-L) \dot{\phi}+L(M(\gamma, \delta))+(\dot{M}(\dot{c}, \dot{d})-M(\dot{c}, \dot{d}))+\dot{E}+\dot{Q}(\dot{\phi})=0 .
$$

Taking the difference with the first equation, we get

$$
\begin{aligned}
\mathcal{L}(\phi-\dot{\bar{\phi}}, c-\dot{c}, d-\dot{d})= & (\dot{L}-L) \dot{\phi}+L(M(\gamma, \delta))+(\dot{M}(\dot{c}, \dot{d})-M(\dot{c}, \dot{d})) \\
& +(\dot{E}-E)+(\dot{Q}(\dot{\phi})-Q(\dot{\phi}))+(Q(\dot{\phi})-Q(\phi)) .
\end{aligned}
$$

Using the arguments we have already used to prove the existence of a solution together with (4.3), we see that there exists $\delta_{3}>0$ such that

$$
\begin{aligned}
\|\phi-\dot{\bar{\phi}}\|_{*}+\|c-\dot{c}\|+\|d-\dot{d}\| \leq & C e^{-\delta_{3} \ell}\left(\sup _{j}\left|\dot{y}_{j}-y_{j}\right|+\sup _{h}\left|\dot{z}_{h}-z_{h}\right|\right) \\
& +C e^{-\delta_{3} \ell}(\|\gamma\|+\|\delta\|)+C e^{-\delta_{3} \ell}\|\overline{\bar{\phi}}-\phi\|_{*} .
\end{aligned}
$$


Moreover, from the definition of $\gamma$ and $\delta$ we also have the estimate

$$
\|\gamma\|+\|\delta\| \leq C\|\phi\|_{*}\left(\sup _{j}\left|\dot{y}_{j}-y_{j}\right|+\sup _{h}\left|\dot{z}_{h}-z_{h}\right|\right),
$$

and hence we conclude that

$$
\|\phi-\dot{\bar{\phi}}\|_{*}+\|c-\dot{c}\|+\|d-\dot{d}\| \leq C e^{-\delta_{3} \ell}\left(\sup _{j}\left|\dot{y}_{j}-y_{j}\right|+\sup _{h}\left|\dot{z}_{h}-z_{h}\right|\right)
$$

provided $\ell$ is chosen large enough. This shows that the solution depends continuously on the parameters defining the points where the copies of $\pm w$ are centered. Indeed, this even proves that the solution is Lipschitz with respect to these parameters.

Let us summarize what we have obtained so far. Given points $y_{j}$ and $z_{h}$ defined in (2.1) and (2.2) and satisfying constraint (2.4), Proposition 4.1 guarantees the existence of a solution $(\phi, c, d)$ of (4.1). Moreover, we have some estimate on the function $\phi$ in the $L^{\infty}$ weighted norm $\|\cdot\|_{*}$ and classical elliptic regularity theory implies that these estimates extend to higher derivatives of $\phi$. The function $u=U+\phi$ will then be the solution of (1.8) we are looking for if we can show that there exists a configuration of the points $y_{j}$ and $z_{h}$ for which the parameters $c_{j}$ and $d_{h}$ are all equal to zero.

In the next section, we find a precise expansion of the parameters $c_{j}$ and $d_{h}$ in terms of the free parameters in the construction (namely $\alpha_{j}, \beta_{h}$ and $\gamma_{h}$ which have been used to define $y_{j}$ and $z_{h}$ ). This expansion is obtained by projecting, in $L^{2}\left(\mathbb{R}^{N}\right)$, equation (4.1) onto the space spanned by $e_{j} \cdot \Xi(\cdot-y)$ for $y \in \Pi$ and $j=1, \ldots, N$, as was already done in the proof of Lemma 3.1. We also explain how to solve the projected problem, and this will complete the proof of Theorem 1.2. Observe that, given the symmetries of the solutions we are looking for, there are obvious relations between $d_{2 n-h}$ and $d_{h}$ for $h=1, \ldots, n$. In particular, $d_{n}$ is collinear to $\mathrm{n}$ and, for $h=1, \ldots, n-1, d_{2 n-h}$ can be expressed in terms of $d_{h}$. Hence the number of equations we have to solve is $2 n+m$, which is also the number of free parameters $\alpha_{1}, \ldots, \alpha_{m+1}, \beta_{1}, \ldots, \beta_{n-1}$ and $\gamma_{1}, \ldots, \gamma_{n}$ we have in our construction.

\section{Projections of the error and the proof of the theorem}

Again, we keep the notations and assumptions of the previous sections. We further assume that $m$ (and $n$ ) are bounded by a constant times $\ell^{A}$ for some $A>0$.

For all $\bar{n} \geq 2$, we define the following $\bar{n} \times \bar{n}$ matrix:

$$
T:=\left(\begin{array}{ccccc}
2 & -1 & 0 & \ldots & 0 \\
-1 & 2 & \ddots & \ddots & \vdots \\
0 & \ddots & \ddots & \ddots & 0 \\
\vdots & \ddots & \ddots & 2 & -1 \\
0 & \ldots & 0 & -1 & 2
\end{array}\right) \in \mathcal{M}_{\bar{n} \times \bar{n}}
$$


In applications, the integer $\bar{n}$ will be equal to $m+1, n$ or $n-1$. It is easy to check that the inverse of $T$ is the matrix whose entries are given by

$$
\left(T^{-1}\right)_{i j}=\min (i, j)-\frac{i j}{\bar{n}+1} .
$$

We define the vectors $S^{\downarrow}$ and $S^{\uparrow}$ by

$$
T S^{\uparrow}:=(1,0, \ldots, 0)^{t} \in \mathbb{R}^{\bar{n}}, \quad T S^{\downarrow}:=(0, \ldots, 0,1)^{t} \in \mathbb{R}^{\bar{n}},
$$

where the superscript ${ }^{t}$ is the transpose so that these are identified with column matrices. We have explicitly

$$
S^{\uparrow}:=\frac{1}{\bar{n}+1}(\bar{n}, \bar{n}-1, \ldots, 2,1)^{t} \quad \text { and } \quad S^{\downarrow}:=\frac{1}{\bar{n}+1}(1,2, \ldots, \bar{n}-1, \bar{n})^{t} .
$$

It will be convenient to write

$$
\alpha:=\left(\alpha_{1}, \ldots, \alpha_{m+1}\right)^{t}, \quad \beta:=\left(\beta_{1}, \ldots, \beta_{n-1}\right)^{t}, \quad \gamma:=\left(\gamma_{1}, \ldots, \gamma_{n}\right)^{t},
$$

where $\alpha_{j}, \beta_{h}$ and $\gamma_{h}$ are the parameters involved in the construction of the points $y_{j}$ and $z_{h}$ which were given in (2.1) and (2.2). As usual, we assume that these parameters satisfy (2.4).

As in the introduction (see (1.6)), we define the interaction function

$$
\Psi(s):=-\int_{\mathbb{R}^{N}} w(\cdot-s e) \operatorname{div}\left(w^{p} e\right) d x
$$

where $e \in \mathbb{R}^{N}$ is a unit vector. The proof of our result is based on the following key lemma:

Lemma 5.1. There exists a constant $C_{N, p}>0$ only depending on $N$ and $p$ such that

$$
\Psi(s)=C_{N, p} e^{-s} s^{-(N-1) / 2}\left(1+\mathcal{O}\left(s^{-1}\right)\right) \quad \text { as } s \rightarrow \infty .
$$

The proof of the above result is standard; we refer to [14] and [12] for details.

Finally, we define the numbers

$$
\kappa:=-(\log \Psi)^{\prime}(\ell) \quad \text { and } \quad \bar{\kappa}:=-(\log \Psi)^{\prime}(\bar{\ell}),
$$

as well as

$$
\lambda_{1}:=1-2 \frac{\bar{\kappa}}{\kappa} \sin \frac{\pi}{k} \quad \text { and } \quad \lambda_{2}:=1+\frac{\bar{\kappa}}{\kappa} \sin \frac{\pi}{k}-\bar{\ell}^{-1} \frac{1}{\kappa} \cot \frac{\pi}{k} \cos \frac{\pi}{k} .
$$

Observe that all these numbers depend on $\ell, \bar{\ell}, m$ and $n$ but they converge to limits as $\ell$ tends to infinity. In fact, according to (1.12), we have

$$
\lim _{\ell \rightarrow \infty} \kappa=\lim _{\ell \rightarrow \infty} \bar{\kappa}=1
$$

and

$$
\lim _{\ell \rightarrow \infty} \lambda_{1}=1-2 \sin \frac{\pi}{k} \quad \text { and } \quad \lim _{\ell \rightarrow \infty} \lambda_{2}=1+\sin \frac{\pi}{k} .
$$

The main result of this section is the following: 
Proposition 5.1. Let $\phi$ be the solution of (4.1) obtained in Proposition 4.1. The coefficients $c_{j}$ and $d_{h}$ are all equal to 0 if and only if the column vectors $\alpha \in \mathbb{R}^{m+1}, \beta \in \mathbb{R}^{n-1}$ and $\gamma \in \mathbb{R}^{n}$ are solutions of the following nonlinear system:

$$
\begin{aligned}
\alpha & =\lambda_{1} \alpha_{1} S^{\uparrow}+\left(\frac{\bar{\kappa}}{\kappa} \beta_{1}+\frac{1}{\kappa} \cot \frac{\pi}{k} \gamma_{1}+\lambda_{2} \alpha_{m+1}\right) S^{\downarrow}+e^{-\delta_{2} \ell} B_{\alpha}+D_{\alpha}, \\
\beta & =-\sin \frac{\pi}{k} \alpha_{m+1} S^{\uparrow}+e^{-\delta_{2} \ell} B_{\beta}+D_{\beta}, \\
\bar{\ell} \gamma & =\cos \frac{\pi}{k} \alpha_{m+1} S^{\uparrow}+\bar{\ell} \gamma_{n} S^{\downarrow}+e^{-\delta_{2} \ell} B_{\gamma}+D_{\gamma},
\end{aligned}
$$

where $\delta_{2}>0, B_{\bullet}:=B_{\bullet}(\ell, m, n ; \alpha, \beta, \gamma)$ denote smooth vector-valued functions whose Taylor expansions in $\alpha, \beta$ and $\gamma$ have coefficients which are uniformly bounded as $\ell \rightarrow \infty$, provided $\alpha, \beta$ and $\gamma$ satisfy (2.4). Moreover, $D_{\bullet}:=D_{\bullet}(\ell, m, n ; \alpha, \beta, \gamma)$ denote smooth vector-valued functions which are quadratic in $\alpha, \beta$ and $\gamma$ as $\ell \rightarrow \infty$.

The proof of this proposition relies on the following technical lemmas. First, using elementary geometry, we find:

Lemma 5.2. The following expansions hold:

$$
\frac{1}{\Psi(\ell)} \int_{\mathbb{R}^{N}} \Xi\left(\cdot-y_{1}\right) E d x=\left(\kappa\left(\alpha_{2}-\alpha_{1}\right)-2 \sin \frac{\pi}{k} \bar{\kappa} \alpha_{1}\right) e_{1}+e^{-\delta_{3} \ell} B+D,
$$

and, for $j=2, \ldots, m$,

$$
\begin{aligned}
\frac{1}{\Psi(\ell)} \int_{\mathbb{R}^{N}} \Xi\left(\cdot-y_{j}\right) E d x= & \kappa\left(\alpha_{j-1}-2 \alpha_{j}+\alpha_{j+1}\right) e_{1}+e^{-\delta_{3} \ell} B+D \\
\frac{1}{\Psi(\ell)} \int_{\mathbb{R}^{N}} \Xi\left(\cdot-y_{m+1}\right) E d x= & \left(\kappa\left(\alpha_{m}-\alpha_{m+1}\right)+\bar{\kappa}\left(\beta_{1}+\sin \frac{\pi}{k} \alpha_{m+1}\right)\right. \\
& \left.+\cot \frac{\pi}{k}\left(\gamma_{1}-\bar{\ell}^{-1} \cos \frac{\pi}{k} \alpha_{m+1}\right)\right) e_{1}+e^{-\delta_{3} \ell} B+D .
\end{aligned}
$$

We also have

$$
\begin{aligned}
\frac{1}{\Psi(\bar{\ell})} \int_{\mathbb{R}^{N}} \Xi\left(\cdot-z_{1}\right) E d x= & \bar{\kappa}\left(2 \beta_{1}-\beta_{2}+\sin \frac{\pi}{k} \alpha_{m+1}\right) \mathrm{t} \\
& +\left(\gamma_{2}-2 \gamma_{1}+\frac{1}{\bar{\ell}} \cos \frac{\pi}{k} \alpha_{m+1}\right) \mathrm{n}+e^{-\delta_{3} \ell} B+D
\end{aligned}
$$

and

$$
\begin{aligned}
\frac{(-1)^{h}}{\Psi(\bar{\ell})} \int_{\mathbb{R}^{N}} \Xi\left(\cdot-z_{h}\right) E d x= & \bar{\kappa}\left(\beta_{h-1}-2 \beta_{h}+\beta_{h+1}\right) \mathrm{t} \\
& -\left(\gamma_{h-1}-2 \gamma_{h}+\gamma_{h+1}\right) \mathrm{n}+e^{-\delta_{3} \ell} B+D
\end{aligned}
$$


for all $h=2, \ldots, n-1$, and finally

$$
\frac{(-1)^{n}}{\Psi(\bar{\ell})} \int_{\mathbb{R}^{N}} \Xi\left(\cdot-z_{n}\right) E d x=2\left(\gamma_{n}-\gamma_{n-1}\right) \mathrm{n}+e^{-\delta_{3} \ell} B+D,
$$

where $\delta_{3}>0, B=B(\ell, m, n ; \alpha, \beta, \gamma)$ denote smooth vector-valued functions (which vary from line to line) whose Taylor expansions in $\alpha, \beta$ and $\gamma$ have coefficients which are uniformly bounded as $\ell \rightarrow \infty$, provided $\alpha, \beta$ and $\gamma$ satisfy (2.4). Moreover, $D=$ $D(\ell, m, n ; \alpha, \beta, \gamma)$ denote smooth vector-valued functions which are quadratic in $\alpha, \beta$ and $\gamma$ as $\ell \rightarrow \infty$.

Proof. Given $y \in \Pi$, we want to estimate $\int_{\mathbb{R}^{N}} \Xi(\cdot-y) E d x$. Observe that, given the structure of $U$ and the fact that $w$ decays exponentially, we can write, using Taylor's expansion,

$$
\begin{aligned}
\int_{\mathbb{R}^{N}} \Xi(\cdot-y) E d x & =\sum_{z \in \Pi_{y}} \epsilon_{z} \int_{\mathbb{R}^{N}} w(\cdot-z) p w^{p-1}(\cdot-y) \Xi(\cdot-y) d x+e^{-\delta_{4} \ell} B \\
& =\sum_{z \in \Pi_{y}} \epsilon_{z} \int_{\mathbb{R}^{N}} w(\cdot-z) p w^{p-1}(\cdot-y) \nabla w(\cdot-y) d x+e^{-\delta_{4} \ell} B \\
& =-\sum_{z \in \Pi_{y}} \epsilon_{z} \Psi(|z-y|) \frac{z-y}{|z-y|}+e^{-\delta_{4} \ell} B
\end{aligned}
$$

where $B$ varies from line to line and is uniformly bounded as $\ell \rightarrow \infty$, and where $\Pi_{y}$ is the set of closest neighbors of $y$ in $\Pi$, that is, points in $\Pi$ whose distance from $y$ is equal to $\ell+\mathcal{O}(1)$. Here $\epsilon_{z}= \pm 1$ according to the sign in front of $w(\cdot-z)$ in the definition of $U$, and $\delta_{4}>1$.

Observe that, in our case, if $z \in \Pi_{y}$, then one can write

$$
z-y=\tilde{\ell} e+a
$$

where $\tilde{\ell} \sim \ell$, e $\in \mathbb{R}^{N}$ satisfies $|\mathrm{e}|=1$ and where $a \in \mathbb{R}^{N}$ is bounded independently of $\ell$. Therefore, we also need an expansion of $\Psi(|\tilde{\ell} e+a|)(\tilde{\ell} e+a) /|\tilde{\ell} e+a|$ as $\tilde{\ell}$ tends to infinity.

Given $e \in \mathbb{R}^{N}$ with $|e|=1$ and $a \in \mathbb{R}^{N}$, we can decompose

$$
a=a^{l l}+a^{\perp},
$$

where $a^{l l}$ is collinear to $e$ and $a^{\perp}$ is orthogonal to e. We claim that

$$
\Psi(|\tilde{\ell} e+a|) \frac{\tilde{\ell} e+a}{|\tilde{\ell} e+a|}=\Psi(\tilde{\ell})\left(e-\tilde{\kappa} a^{\|}+\tilde{\ell}^{-1} a^{\perp}+\mathcal{O}\left(|a|^{2}\right)\right)
$$

as $\tilde{\ell} \rightarrow \infty$, where $\tilde{\kappa}:=-(\log \Psi)^{\prime}(\tilde{\ell})$. This expansion follows at once from the expansion of $\Psi$. Indeed,

$$
|\tilde{\ell} e+a|=\tilde{\ell}\left(1+\tilde{\ell}^{-1}(e \cdot a)+\mathcal{O}\left(\tilde{\ell}^{-2}|a|^{2}\right)\right),
$$


and hence

$$
\Psi(|\tilde{\ell} e+a|)=\Psi(\tilde{\ell})+\Psi^{\prime}(\tilde{\ell}) e \cdot a+\Psi(\tilde{\ell}) \mathcal{O}\left(|a|^{2}\right)
$$

Similarly, we can expand

$$
\frac{\tilde{\ell} e+a}{|\tilde{\ell} e+a|}=\tilde{\ell}\left(e-\tilde{\ell}^{-1}(e \cdot a) e+\tilde{\ell}^{-1} a+\mathcal{O}\left(\tilde{\ell}^{-2}|a|^{2}\right)\right) .
$$

The claim then follows at once.

This expansion, together with (5.5), gives the expansion of $\int_{\mathbb{R}^{N}} \Xi(\cdot-y) E d x$ in terms of the closest neighbors of $y$. Therefore, to complete the proof of the lemma, it is enough to identify, in each case, the closest neighbors of the point $y \in \Pi$ we are considering.

We recall that $\Gamma$ denotes the symmetry with respect to the $x_{2}=0$ hyperplane and $R_{k}$ is the rotation of angle $2 \pi / k$ in the $\left(x_{1}, x_{2}\right)$-plane. We now collect a few useful identities. First, recall that we have defined

$$
\mathrm{t}:=-\sin \frac{\pi}{k} e_{1}+\cos \frac{\pi}{k} e_{2} \quad \text { and } \quad \mathrm{n}:=\cos \frac{\pi}{k} e_{1}+\sin \frac{\pi}{k} e_{2} .
$$

It is easy to check that

$$
R_{k} e_{1}-e_{1}=2 \sin \frac{\pi}{k} t
$$

We define

$$
t^{*}:=\Gamma \mathrm{t} \quad \text { and } \quad \mathrm{n}^{*}:=\Gamma \mathrm{n} .
$$

Observe that

$$
t+t^{*}=-2 \sin \frac{\pi}{k} e_{1} \text { and } n^{*}+n=2 \cos \frac{\pi}{k} e_{1}
$$

Proof of the first expansion. In $\Pi$, the closest neighbors of $y_{1}$ are $y_{2}, R_{k} y_{1}$ and $R_{k}^{-1} y_{1}$. It follows from the definition of the points in $\Pi$ as well as the definition of $\bar{\ell}$ given in (1.24) that

$$
y_{2}-y_{1}=\ell e_{1}+\left(\alpha_{2}-\alpha_{1}\right) e_{1}, \quad R_{k} y_{1}-y_{1}=\bar{\ell} t+2 \sin \frac{\pi}{k} \alpha_{1} t
$$

and

$$
R_{k}^{-1} y_{1}-y_{1}=\bar{\ell} t^{*}+2 \sin \frac{\pi}{k} \alpha_{1} t^{*} .
$$

Using the expansion (5.6), we get

$$
\begin{aligned}
\int_{\mathbb{R}^{N}} \Xi\left(\cdot-y_{1}\right) E d x= & -\left(\Psi(\ell) e_{1}+\Psi(\bar{\ell})\left(\mathrm{t}+\mathrm{t}^{*}\right)\right)+\Psi(\ell) \kappa\left(\alpha_{2}-\alpha_{1}\right) e_{1} \\
& +\Psi(\bar{\ell}) 2 \sin \frac{\pi}{k} \bar{\kappa} \alpha_{1}\left(\mathrm{t}+\mathrm{t}^{*}\right)+e^{-\delta_{5} \ell} B+\Psi(\ell) D,
\end{aligned}
$$

where $\delta_{5}>1$. The first expansion in Lemma 5.2 follows from the fact that $\ell$ and $\bar{\ell}$ are related by (1.24) together with (5.10).

Proof of the second expansion. In $\Pi$, the closest neighbors of $y_{j}$ are $y_{j-1}$ and $y_{j+1}$. Observe that, thanks to $k \geq 7$, the distance between $y_{m}$ and $z_{1}$ can be estimated by 
$2 \sin \theta \ell+\mathcal{O}(1)$ where $\theta=\frac{\pi}{4}-\frac{\pi}{2 k}>\frac{\pi}{6}$ and hence is much larger than $\ell+\mathcal{O}(1)$. Therefore, the closest neighbors of $y_{m}$ are again $y_{m-1}$ and $y_{m+1}$. Since

$$
y_{j+1}-y_{j}=\ell e_{1}+\left(\alpha_{j+1}-\alpha_{j}\right) e_{1} \quad \text { and } \quad y_{j-1}-y_{j}=-\ell e_{1}+\left(\alpha_{j-1}-\alpha_{j}\right) e_{1},
$$

we can make use of (5.6) to conclude that

$$
\int_{\mathbb{R}^{N}} \Xi\left(\cdot-y_{j}\right) E d x=\Psi(\ell) \kappa\left(\alpha_{j-1}-2 \alpha_{j}+\alpha_{j+1}\right) e_{1}+e^{-\delta_{5} \ell} B+\Psi(\ell) d,
$$

where $\delta_{5}>1$, and this completes the proof of the second expansion.

Proof of the third expansion. The closest neighbors of $y_{m+1}$ in $\Pi$ are $y_{m}, z_{1}$ and $R_{k}^{-1} z_{2 n-1}=\Gamma z_{1}$. We have

$$
\begin{aligned}
y_{m}-y_{m+1} & =-\ell e_{1}+\left(\alpha_{m}-\alpha_{m+1}\right) e_{1}, \\
z_{1}-y_{m+1} & =\bar{\ell} \mathrm{t}+\left(\beta_{1}+\sin \frac{\pi}{k} \alpha_{m+1}\right) \mathrm{t}+\left(\bar{\ell} \gamma_{1}-\cos \frac{\pi}{k} \alpha_{m+1}\right) \mathrm{n}, \\
\Gamma z_{1}-y_{m+1} & =\bar{\ell} \mathrm{t}^{*}+\left(\beta_{1}+\sin \frac{\pi}{k} \alpha_{m+1}\right) \mathrm{t}^{*}+\left(\bar{\ell} \gamma_{1}-\cos \frac{\pi}{k} \alpha_{m+1}\right) \mathrm{n}^{*} .
\end{aligned}
$$

Making use of (5.6), we get

$$
\begin{aligned}
\int_{\mathbb{R}^{N}} \Xi\left(\cdot-y_{m+1}\right) E d x= & \left(\Psi(\ell) e_{1}+\Psi(\bar{\ell})\left(\mathrm{t}^{*}+\mathrm{t}\right)\right)+\Psi(\ell) \kappa\left(\alpha_{m}-\alpha_{m+1}\right) e_{1} \\
& -\Psi(\bar{\ell}) \bar{\kappa}\left(\beta_{1}+\sin \frac{\pi}{k} \alpha_{m+1}\right)\left(\mathrm{t}^{*}+\mathrm{t}\right) \\
& +\Psi(\bar{\ell})\left(\gamma_{1}-\bar{\ell}^{-1} \cos \frac{\pi}{k} \alpha_{m+1}\right)\left(\mathrm{n}^{*}+\mathrm{n}\right)+e^{-\delta_{5} \ell} B+\Psi(\ell) d,
\end{aligned}
$$

where $\delta_{5}>1$. One should be careful that the copies of $w$ come with positive signs at $y_{m+1}$ and $y_{m}$, but with negative signs at $z_{1}$ and $\Gamma z_{1}$. The formula follows at once from (5.10).

Proof of the fourth expansion. The closest neighbors of $z_{1}$ in $\Pi$ are $y_{m+1}$ and $z_{2}$. We can write

$$
\begin{aligned}
y_{m+1}-z_{1} & =-\bar{\ell} t-\left(\beta_{1}+\sin \frac{\pi}{k} \alpha_{m+1}\right) \mathrm{t}-\left(\bar{\ell} \gamma_{1}-\cos \frac{\pi}{k} \alpha_{m+1}\right) \mathrm{n}, \\
z_{2}-z_{1} & =\bar{\ell} t+\left(\beta_{2}-\beta_{1}\right) t+\bar{\ell}\left(\gamma_{2}-\gamma_{1}\right) \mathrm{n} .
\end{aligned}
$$

Arguing as above, we get

$$
\begin{aligned}
\int_{\mathbb{R}^{N}} \Xi\left(\cdot-z_{1}\right) E d x= & \Psi(\bar{\ell}) \bar{\kappa}\left(2 \beta_{1}-\beta_{2}+\sin \frac{\pi}{k} \alpha_{m+1}\right) \mathrm{t} \\
& +\Psi(\bar{\ell})\left(\gamma_{2}-2 \gamma_{1}+\ell^{-1} \cos \frac{\pi}{k} \alpha_{m+1}\right) \mathrm{n}+e^{-\delta_{5} \ell} B+\Psi(\ell) d,
\end{aligned}
$$


where $\delta_{5}>1$. Again, one should be careful that the copies of $w$ come with alternating signs. The proof of the fourth expansion then follows at once.

Proof of the fifth and sixth expansions. For $h=2, \ldots, n$, we have

$$
\begin{aligned}
& z_{h-1}-z_{h}=-\bar{\ell} t+\left(\beta_{h-1}-\beta_{h}\right) t+\bar{\ell}\left(\gamma_{h-1}-\gamma_{h}\right) \mathrm{n}, \\
& z_{h+1}-z_{h}=\bar{\ell} t+\left(\beta_{h+1}-\beta_{h}\right) t+\bar{\ell}\left(\gamma_{h+1}-\gamma_{h}\right) \mathrm{n} .
\end{aligned}
$$

Applying (5.6), we conclude that

$$
\begin{aligned}
(-1)^{h} \int_{\mathbb{R}^{N}} \Xi\left(\cdot-z_{h}\right) E d x= & \Psi(\bar{\ell}) \bar{\kappa}\left(\beta_{h-1}-2 \beta_{h}+\beta_{h+1}\right) \mathrm{t} \\
& -\Psi(\bar{\ell})\left(\gamma_{h-1}-2 \gamma_{h}+\gamma_{h+1}\right) \mathrm{n}+e^{-\delta_{5} \ell} B+\Psi(\ell) d,
\end{aligned}
$$

where $\delta_{5}>1$. Again, one should be careful that the copies of $w$ come with alternating signs. This completes the proof of the fifth expansion. The sixth expansion follows from similar considerations.

The next result is easier to get:

Lemma 5.3. The following expansions hold:

$$
\int_{\mathbb{R}^{N}} \Xi(\cdot-y) L \phi d x=\Psi(\ell) e^{-\delta_{3} \ell} B, \quad \int_{\mathbb{R}^{N}} \Xi(\cdot-y) Q(\phi) d x=\Psi(\ell) e^{-\delta_{3} \ell} B,
$$

where $\delta_{3}>0$ and $B=B(\ell, m, n ; \alpha, \beta, \gamma)$ denote (various) smooth vector-valued functions whose Taylor expansions in $\alpha, \beta$ and $\gamma$ have coefficients uniformly bounded as $\ell \rightarrow \infty$, provided $\alpha, \beta$ and $\gamma$ satisfy (2.4).

Proof. The key point is to prove that both quantities tend to 0 much faster than $e^{-\ell}$ as $\ell$ tends to infinity. Both estimates rely on the fact that, by construction, the solution $\phi$ defined in Proposition 4.1 satisfies

$$
\|\phi\|_{*} \leq C e^{-\delta_{1} \ell}
$$

and, as mentioned right after the statement of that proposition, it is possible to choose $\eta<0$ in the definition of the $\|\cdot\|_{*}$-norm in such a way that $\delta_{1}>1 / 2$.

Now, observe that

$$
\int_{\mathbb{R}^{N}} \Xi(\cdot-y) L \phi d x=\int_{\mathbb{R}^{N}} \phi L(\Xi(\cdot-y)) d x .
$$

Taking the above remark into consideration, the proof of the first expansion follows the line of the proof of Lemma 3.1.

The second expansion follows from the proof of the estimates in Proposition 4.1.

Proof of Proposition 5.1. Recall that, as $\ell$ tends to infinity,

$$
\int_{\mathbb{R}^{N}}\left(\mathrm{e}_{j} \cdot \Xi(\cdot-y)\right)\left(\mathrm{e}_{i} \cdot \Xi(\cdot-z)\right) d x=\frac{1+o(1)}{N} \int_{\mathbb{R}^{N}}|\nabla w|^{2} d x
$$


if $i=j$ and $y=z \in \Pi$, and is equal to 0 otherwise, as was already mentioned in the proof of Lemma 3.1.

Now, we use the identity

$$
\int_{\mathbb{R}^{N}}(\mathcal{L}(\phi, c, d)+E+Q(\phi))\left(\mathrm{e}_{i} \cdot \Xi(\cdot-y)\right) d x=0,
$$

so that, thanks to the above remark, all $c_{i}$ and $d_{h}$ are zero if and only if

$$
\int_{\mathbb{R}^{N}}(L \phi+E+Q(\phi))\left(e_{i} \cdot \Xi(\cdot-y)\right) d x=0 \quad \text { for all } y \in \Pi \text { and } i=1, \ldots, N \text {. }
$$

Using the previous lemmas, it is easy to check that this reduces to the solvability of a nonlinear system in $\alpha, \beta$ and $\gamma$ which can be written in the form

$$
\begin{aligned}
& \left\{\begin{array}{l}
2 \alpha_{1}-\alpha_{2}=\left(1-2 \frac{\bar{\kappa}}{\kappa} \sin \frac{\pi}{k}\right) \alpha_{1}+e^{-\delta_{3} \ell} B+D, \\
-\alpha_{j-1}+2 \alpha_{j}-\alpha_{j+1}=e^{-\delta_{3} \ell} B+D \text { for } j=2, \ldots, m, \\
-\alpha_{m}+2 \alpha_{m+1}=\frac{\bar{\kappa}}{\kappa} \beta_{1}+\left(1+\frac{\bar{\kappa}}{\kappa} \sin \frac{\pi}{k}\right) \alpha_{m+1} \\
\quad+\frac{1}{\kappa} \cot \frac{\pi}{k}\left(\gamma_{1}-\bar{\ell}^{-1} \cos \frac{\pi}{k} \alpha_{m+1}\right)+e^{-\delta_{3} \ell} B+D,
\end{array}\right. \\
& \left\{\begin{array}{l}
2 \beta_{1}-\beta_{2}=-\sin \frac{\pi}{k} \alpha_{m+1}+e^{-\delta_{3} \ell} B+D, \\
-\beta_{h-1}+2 \beta_{h}-\beta_{h+1}=e^{-\delta_{3} \ell} B+D \text { for } h=2, \ldots, n-2, \\
-\beta_{n-2}+2 \beta_{n-1}=e^{-\delta_{3} \ell} B+D,
\end{array}\right.
\end{aligned}
$$

and

$$
\left\{\begin{array}{l}
2 \gamma_{1}-\gamma_{2}=\bar{\ell}^{-1} \cos \frac{\pi}{k} \alpha_{m+1}+e^{-\delta_{3} \ell} B+D \\
-\gamma_{h-1}+2 \gamma_{h}-\gamma_{h+1}=e^{-\delta_{3} \ell} B+D \text { for } h=2, \ldots, n-1, \\
-\gamma_{n-1}+2 \gamma_{n}=\gamma_{n}+e^{-\delta_{3} \ell} B+D
\end{array}\right.
$$

One recognizes immediately the action of matrices of the form $T$, for $\bar{n}$ equal to $m+1$, $n-1$ or $n$, on the left hand side of these equations. This system can then be put in the desired form using the inverses of the matrices $T$.

Observe that we implicitly use the fact that the integers $n$ and $m$ are bounded by $\ell^{A}$ for some fixed $A>0$, so that the norms of the inverses of the matrices $T$ grow at most polynomially in $\ell$ and this can easily be absorbed since the error tends to 0 exponentially fast in $\ell$.

We now explain how (5.4) can be solved. We claim that this system is equivalent to

$$
\left\{\begin{array}{l}
\alpha=e^{-\tilde{\delta}_{2} \ell} B+D \\
\beta=e^{-\tilde{\delta}_{2} \ell} B+D \\
\gamma=e^{-\tilde{\delta}_{2} \ell} B+D
\end{array}\right.
$$


where $\tilde{\delta}_{2}>0$ and $B=B(\ell, m, n ; \alpha, \beta, \gamma)$ and $D=D(\ell, m, n ; \alpha, \beta, \gamma)$ satisfy the usual assumptions.

Observe that the system (5.4) is almost of the correct form. Below, both $\tilde{\delta}_{2}>0$ and the nonlinear functions $B=B(\ell, m, n ; \alpha, \beta, \gamma)$ and $D=D(\ell, m, n ; \alpha, \beta, \gamma)$ may change from line to line but they satisfy the usual assumptions. In fact, using the second and third equations together with the expressions for $S^{\uparrow}$ and $S^{\downarrow}$ one checks that $\gamma_{1}, \beta_{1}$ and $\gamma_{n}$ can be expressed in terms of $\alpha_{m+1}$ and lower order terms. More precisely, we get

$$
\left\{\begin{aligned}
\beta_{1} & =-\frac{n-1}{n} \sin \frac{\pi}{k} \alpha_{m+1}+e^{-\tilde{\delta}_{2} \ell} B+D \\
\bar{\ell} \gamma_{1} & =\cos \frac{\pi}{k} \alpha_{m+1}+e^{-\tilde{\delta}_{2} \ell} B+D \\
\bar{\ell} \gamma_{n} & =\cos \frac{\pi}{k} \alpha_{m+1}+e^{-\tilde{\delta}_{2} \ell} B+D
\end{aligned}\right.
$$

Hence we compute

$$
\frac{\bar{\kappa}}{\kappa} \beta_{1}+\frac{1}{\kappa} \cot \frac{\pi}{k} \gamma_{1}+\lambda_{2} \alpha_{m+1}=\left(1+\frac{1}{n} \frac{\bar{\kappa}}{\kappa} \sin \frac{\pi}{k}\right) \alpha_{m+1}+e^{-\tilde{\delta}_{2} \ell} B+D .
$$

If we insert these in the first equation, we are left to solve a coupled system in $\alpha_{1}$ and $\alpha_{m+1}$ which reads

$$
\left\{\begin{array}{c}
\left(1+2(m+1) \frac{\bar{\kappa}}{\kappa} \sin \frac{\pi}{k}\right) \alpha_{1}-\left(1+\frac{1}{n} \frac{\bar{\kappa}}{\kappa} \sin \frac{\pi}{k}\right) \alpha_{m+1}=e^{-\tilde{\delta}_{2} \ell} B+D, \\
-\left(1-2 \frac{\bar{\kappa}}{\kappa} \sin \frac{\pi}{k}\right) \alpha_{1}+\left(1-\frac{1}{n}(m+1) \frac{\bar{\kappa}}{\kappa} \sin \frac{\pi}{k}\right) \alpha_{m+1}=e^{-\tilde{\delta}_{2} \ell} B+D .
\end{array}\right.
$$

This system can be solved provided $D_{0}$, the determinant of the 2 by 2 system on the left hand side, is nonzero. But

$$
D_{0}=\frac{\bar{\kappa}}{\kappa} \sin \frac{\pi}{k} \frac{m+2}{n}\left(2 n-1-2 m \sin \frac{\pi}{k} \frac{\bar{\kappa}}{\kappa}\right) .
$$

Using (1.12) together with (1.17), we conclude that

$$
D_{0}=\frac{\bar{\kappa}}{\kappa^{2}} \sin \frac{\pi}{k} \frac{m+2}{n}(2 n-1)\left(\frac{\ell-\bar{\ell}}{\ell}+\mathcal{O}\left(\ell^{-2}\right)\right),
$$

which, thanks to (1.29), is certainly bounded from below by some constant times $m / \ell$ for all $\ell$ large enough. This completes the proof of the claim.

Using Browder's fixed point theorem, one can now prove

Lemma 5.4. There exist $C, \ell_{0}>0$ such that, for all $\ell \geq \ell_{0}$, there exists a solution of (5.11) such that

$$
\|\alpha\|+\|\beta\|+\|\gamma\| \leq C e^{-\tilde{\delta}_{2} \ell},
$$

where, as usual, the norm of a vector is defined to be the sup norm. 
The proof of this last lemma is standard and left to the reader and follows from the properties of $B_{\bullet}$ and $D_{\bullet}$ in Proposition 5.1. Observe that, with some more care, one can prove that the solution in Proposition 4.1 depends smoothly on the parameters and then (increasing the value of $\ell_{0}$ if necessary) one can use a fixed point theorem for contraction mappings to prove Lemma 5.4. This has the advantage of giving local uniqueness for the solution of (5.11), which in turn shows the unique (local) solvability of the nonlinear equation once the parameters $m, n$ and $\ell, \bar{\ell}$ solutions of (1.17) and (1.24) are fixed.

This last result completes the proof of Theorem 1.2.

\section{Appendix}

To complete the paper, we now explain how to formally justify the constraint we impose on the choice of the parameters $\ell$ and $\bar{\ell}$. Let us recall that if $u$ is a solution of (1.8) then

$$
\operatorname{div}\left((a \cdot \nabla u) \nabla u-\frac{1}{2}\left(|\nabla u|^{2}+u^{2}\right) a+\frac{1}{p+1}|u|^{p+1} a\right)=0
$$

for any fixed vector $a \in \mathbb{R}^{N}$ (just multiply (1.8) by $a \cdot \nabla u$ and use simple manipulations). In particular, the divergence theorem implies that, for any smooth domain $\Omega \subset \mathbb{R}^{N}$, the vector

$$
Y(u, \Omega):=\int_{\partial \Omega}\left((\nabla u \cdot v) \nabla u-\frac{1}{2}\left(|\nabla u|^{2}+u^{2}\right) v+\frac{1}{p+1}|u|^{p+1} v\right) d \sigma
$$

is equal to 0 . Here $v$ is the outward unit normal vector field to $\partial \Omega$. We hope that a function of the form

$$
U=w+\sum_{i} \epsilon_{i} w\left(\cdot-z_{i}\right)+\mathcal{O}\left(e^{-3 \ell / 2}\right)
$$

is, in the ball $B_{\ell / 2}$ of radius $\ell / 2$ centered at the origin, close to a genuine solution of (1.8), where $\epsilon_{i} \in\{ \pm 1\}$ and where the points $z_{i}$ have the property that

$$
\left|z_{i}\right|=\ell+\mathcal{O}(1)
$$

If this intuition is correct, then the associated vector $Y\left(U, B_{\ell / 2}\right)$ should be reasonably close to 0 as $\ell$ tends to $\infty$. But a computation shows that

$$
Y\left(U, B_{\ell / 2}\right)=\sum_{i} \epsilon_{i} \Psi\left(\left|z_{i}\right|\right) \frac{z_{i}}{\left|z_{i}\right|}+\mathcal{O}\left(e^{-\delta \ell}\right)
$$

for some $\delta>1$, as $\ell$ tends to $+\infty$. Therefore, in order for the construction to be successful, it is reasonable to ask that

$$
\sum_{i} \epsilon_{i} \Psi\left(\left|z_{i}\right|\right) \frac{z_{i}}{\left|z_{i}\right|}=0
$$

This is precisely the balancing condition we were referring to. Applying this to the approximate solution $\stackrel{O}{U}_{\text {at }}$ the points $y_{1}$ and $y_{m+1}$ leads to (1.24). 
Acknowledgments. The authors would like to thank the referees for valuable comments which helped to improve some of the arguments of the paper. This work has been partly supported by the contract C05E05 from the ECOS-CONICYT. The research of the first author has been partly supported by Fondecyt Grant 1120151 and CAPDE-Anillo ACT-125, Chile. The second author is partially supported by the ANR-08-BLANC-0335-01 grant. The research of the third author is supported by an Earmarked Grant from RGC of Hong Kong, Oversea Joint Grant of NSFC, and a direct grant from CUHK.

\section{References}

[1] Bartsch, T., Willem, M.: Infinitely many nonradial solutions of a Euclidean scalar field equation. J. Funct. Anal. 117, 447-460 (1993) Zbl 0790.35021 MR 1244943

[2] Bartsch, T., Willem, M.: Infinitely many radial solutions of a semilinear elliptic problem on $\mathbb{R}^{N}$. Arch. Rat. Mech. Anal. 124, 261-276 (1993) Zbl 0790.35020 MR 1237913

[3] Berestycki, H., Lions, P.-L.: Nonlinear scalar field equations, I. Existence of a ground state. Arch. Rat. Mech. Anal. 82, 313-345 (1981) Zbl 0533.35029 MR 0695535

[4] Berestycki, H., Lions, P.-L.: Nonlinear scalar field equations, II. Arch. Rat. Mech. Anal. 82, 347-375 (1981) Zbl 0556.35046 MR 0695536

[5] Conti, M., Merizzi, L., Terracini, S.: Radial solutions of superlinear problems in $\mathbb{R}^{N}$. Arch. Rat. Mech. Anal. 153, 291-316 (2000) Zbl 0961.35043 MR 1773818

[6] del Pino, M., Kowalczyk, M., Pacard, F., Wei, J.: The Toda system and multiple-end solutions of autonomous planar elliptic problems. Adv. Math. 224, 1462-1516 (2010) Zbl 1197.35114 MR 2646302

[7] Gidas, B., Ni, W. M., Nirenberg, L.: Symmetry of positive solutions of nonlinear elliptic equations in $\mathbb{R}^{n}$. In: Mathematical Analysis and Applications, Part A, Adv. Math. Suppl. Stud. 7A, Academic Press, New York, 369-402 (1981) Zbl 0469.35052 MR 0634248

[8] Jleli, M., Pacard, F.: An end-to-end construction for compact constant mean curvature surfaces. Pacific J. Math. 221, 81-108 (2005) Zbl 1110.53043 MR 2194146

[9] Kapouleas, N.: Compact constant mean curvature surfaces in Euclidean three-space. J. Differential Geom. 33, 683-715 (1991) Zbl 0727.53063 MR 1100207

[10] Kwong, M. K.: Uniqueness of positive solutions of $\Delta u-u+u^{p}=0$ in $\mathbb{R}^{n}$. Arch. Rat. Mech. Anal. 105, 243-266 (1989) Zbl 0676.35032 MR 0969899

[11] Kwong, M. K., Zhang, L.: Uniqueness of the positive solution of $\Delta u+f(u)=0$ in an annulus. Differential Integral Equations 4, 583-599 (1991) Zbl 0724.34023 MR 1097920

[12] Lin, F. H., Ni, W. M., Wei, J. C.: On the number of interior peak solutions for a singularly perturbed Neumann problem. Comm. Pure Appl. Math. 60, 252-281 (2007) Zbl 1170.35424 MR 2275329

[13] Lorca, S., Ubilla, P.: Symmetric and nonsymmetric solutions for an elliptic equation on $\mathbb{R}^{N}$. Nonlinear Anal. 58, 961-968 (2004) Zbl 1127.35015 MR 2086066

[14] Malchiodi, A.: Some new entire solutions of semilinear elliptic equations on $\mathbb{R}^{n}$. Adv. Math. 221, 1843-1909 (2009) Zbl 1178.35186 MR 2522830

[15] Ni, W. M., Takagi, I.: Locating the peaks of least-energy solutions to a semilinear Neumann problem. Duke Math. J. 70, 247-281 (1993) Zbl 0796.35056 MR 1219814

[16] Struwe, M.: Multiple solutions of differential equations without the Palais-Smale condition. Math. Ann. 261, 399-412 (1982) Zbl 0506.35034 MR 0679798

[17] Wei, J. C., Yan, S.: Infinitely many positive solutions for the nonlinear Schrödinger equations in $\mathbb{R}^{N}$. Calc. Var. Partial Differential Equations 37, 423-439 (2010) Zbl 1189.35106 MR 2592980 